\title{
Variation of the angular anisotropy in resonant Auger decay along the $\mathrm{Mg} 2 p \rightarrow n l$ excitations
}

\author{
U. Hergenhahn, ${ }^{1}$ S. B. Whitfield, ${ }^{2}$ J. Tulkki, ${ }^{3}$ F. Heiser, ${ }^{1}$ N. M. Kabachnik, ${ }^{4}$ B. Langer, ${ }^{1}$ and U. Becker ${ }^{1}$ \\ ${ }^{1}$ Fritz-Haber-Institut der Max-Planck-Gesellschaft, D-14195 Berlin, Germany \\ ${ }^{2}$ Department of Physics, University of South Alabama, Mobile, Alabama 36688-0002 \\ ${ }^{3}$ Optoelectronics Laboratory, Helsinki University of Technology, SF-02150 Espoo, Finland \\ ${ }^{4}$ Institute of Nuclear Physics, Moscow State University, Moscow 119899, Russia
}

(Received 20 September 1996)

\begin{abstract}
Energy- and angle-resolved resonant Auger spectra were recorded on the first $12 \mathrm{Mg} 2 p \rightarrow n l$ resonances. Utilization of a high-resolution monochromator has allowed us to selectively excite resonant states which could not be resolved in earlier electron spectrometry measurements. The branching ratios and values of the angular anisotropy parameter $\beta$ were extracted for all resonances. The measurements provide a first basis for a systematic test of the spectator model for the angular anisotropy. Our experimental values are compared with $a b$ initio multiconfiguration Dirac-Fock calculations. The sensitivity of the calculations to the configuration basis size is discussed in detail. [S1050-2947(97)05903-9]
\end{abstract}

PACS number(s): 32.80.Dz, 32.80.Hd, 31.25.Jf

\section{INTRODUCTION}

When an inner-shell electron is excited to a Rydberg state, for example in photoabsorption, the following nonradiative decay of the highly excited atom is usually referred to as a "resonant Auger decay." It is a particular case of autoionization. This entails that besides the resonant pathway a second, direct pathway to the final ionic state of resonant Auger decay exists. In many cases of practical interest, however, the direct transition to the final ionic state is negligible. Thus, no interference between the two channels occurs and the resonant Auger decay can be considered a two-step process.

Resonant Auger decay is typically classified as participator or spectator, depending on whether the excited Rydberg electron is involved in the decay or not. During spectator decay, the excited Rydberg electron can change its principal quantum number. When the principal quantum number of the Rydberg electron is smaller (larger) in the final state than in the intermediate state, shakedown (shakeup) is said to have occurred. We refer to a pure spectator transition as that in which the principal quantum number of the excited Rydberg electron remains unchanged following decay.

The interpretation of resonant Auger spectra has often been carried out within the framework of the "spectator model." Most generally this approach expresses the intuitively appealing hypothesis that the resonantly excited electron causes only a small disturbance to the patterns observed in the normal Auger decay of the same core hole. The spectator model has been widely used for the assignment of resonant Auger lines and the interpretation of relative decay intensities. In any case, however, the outer electron adds additional angular momentum to the decaying system. This entails that its influence on the angular anisotropy can never be neglected, once the energy splitting due to the outer electron-core interaction is observed. Several experimental and theoretical studies on noble gases confirming this assertion have lately been reviewed [1,2].

Despite the numerous investigations carried out so far, little seems to be known about the systematics of the angular anisotropy in resonant Auger decay. Partly this is because in the past many studies focused on one of the noble gases. Resonant Auger decay of these systems preferentially leaves the singly charged ion in a ( 2 hole +1 outer electron) final state after the decay process. Due to the multitude of possible couplings between the pertinent open shells, the Auger spectra are often difficult to disentangle.

A system, for which the resonant Auger decay results in simpler final states should therefore provide a better testing ground especially for the dependence of the angular anisotropy in resonant Auger decay on the excitation process. An ideal candidate which meets this criterion is $\mathrm{Mg}$ with the ground state configuration $[\mathrm{Ne}] 3 s^{2}\left({ }^{1} S_{0}\right)$, where $[\mathrm{Ne}] \mathrm{de}-$ notes a core configuration corresponding to that of neon. The $2 p$ excitations of this element decay to final states consisting of a single electron outside of a closed-shell neonlike core configuration,

$$
\mathrm{Mg}: 2 p^{6} 3 s^{2}+h \nu \rightarrow 2 p^{5} 3 s^{2} n l \rightarrow 2 p^{6} n^{\prime} l^{\prime} .
$$

Therefore, the splitting of lines due to different couplings between the core and excited electron in the final state is absent. This greatly facilitates the analysis of the spectra.

We report in this paper results of angular distribution measurements of autoionizing electrons on the first twelve $\operatorname{Mg} 2 p$ resonances, ranging from $2 p_{3 / 2} \rightarrow 4 s$ to $2 p_{3 / 2} \rightarrow 6 d$. Linearly polarized synchrotron radiation was used for the excitation. To interpret the observed angular distributions theoretically, we have performed extensive multiconfiguration Dirac-Fock (MCDF) calculations for the first six resonances. We have given a brief account of selected results on this issue in Ref. [3]. In particular, we have reported about a "dynamic" suppression of autoionization amplitudes for some decays, similar to a Cooper minimum in photoionization.

The purpose of the present paper is threefold: (i) to present the complete experimental results of our earlier study [3]; (ii) to present high resolution measurements, which allow us to distinguish between neighboring $n s, m d$ channels 
in the excitation and the decay spectra, that were not resolved in previous studies $[3,4]$; and (iii) to delineate our theoretical results in detail.

To our knowledge, this paper is the first experimental state-by-state study of the excitation dependence of the angular anisotropy in resonant Auger decay.

The $2 p$ resonances in atomic $\mathrm{Mg}$ have been observed in total absorption measurements by several authors [5-7]. Four distinct Rydberg series converging to the two thresholds for $\mathrm{Mg} 2 p_{3 / 2}$ and $2 p_{1 / 2}$ photoionization at $57.547 \mathrm{eV}$ and $57.825 \mathrm{eV}$ [5] have been found. Autoionization lines from the pertinent $\mathrm{Mg} 2 p^{-1} n l$ excited states were first observed after electron impact excitation of magnesium vapor [8]. Electron spectroscopy of selectively excited resonances was only possible after the advent of synchrotron radiation. A detailed study of the branching ratios into the different ionic states on several resonances was reported by Whitfield et al. [4]. On the theoretical side a many-body perturbation theory study of $\mathrm{Mg} 3 s$ photoionization including several resonances in the $2 p$ excitation region was performed by Altun [9]. Whitfield et al. [10] applied a resonant scattering theory formulation to understand the anomalous behavior of resonant Auger decay in $\mathrm{Mg}$ following excitation to a high lying Rydberg orbital. To this end they systematically calculated the probabilities for shakeup, pure spectator decay, and shakedown in dependence of $n$. Besides that, they corrected the assignment of the high lying excitation under study. Another study about the systematics of shake transitions using hydrogenic wave functions has recently been published by Armen [11].

In heavier alkaline-earth metals the outermost $n s^{2}$ shell is distorted by interaction with $(n-1) d^{2}$ and $n s(n-1) d$ configurations. For that reason, the excitation spectra for electrons of $p$ symmetry into an outer valence or Rydberg state are considerably more complicated than for the $2 p$ excitations of $\mathrm{Mg}$. Results have been published for the $2 p$ excitations in $\mathrm{Ca}$ [12-15], the $3 p$ excitations in $\mathrm{Ca}$ [16], and the $4 p$ excitations in $\mathrm{Sr}$ [17]. Angle resolved studies of some autoionization lines on the $\mathrm{Ca} 3 p-3 d$ [18] and $\mathrm{Sr} 4 p-4 d$ [19] giant resonances covered only the most intense excitations and final states. Very recently Ueda et al. [20] studied the spectator decay of the $3 p^{5} 4 s^{2}\left({ }^{2} P\right) n d$ excited states in $\mathrm{Ca}$ along the pertinent Rydberg series and above the threshold for ionization of the $n d$ excited electron. These authors were able to show that the kinetic energy of the spectator Auger electrons follows a linear dispersion law. The slope of the kinetic energy curve was derived from a simple quantum defect argument. Shakeup of the outer electron was found to be decisive for the correct explanation of the slope. Moreover, in the case studied by these authors the resonant Auger anisotropy was found to evolve smoothly into the anisotropy of normal Auger decay above threshold. However, their study lacked the resolution to isolate single members of the Rydberg excitation series and no excitations into symmetries other than $n d$ were investigated.

The organization of this work is as follows. In Sec. II we explain the experimental setup and our methods of data analysis. We then shortly review some predictions of the spectator model for the angular anisotropy of resonant Auger decay, as we will need these concepts in the discussion of the experimental results. This is followed by a delineation of our theoretical model. In Secs. IV A and IV B we summarize the experimental results for excitations to $n s$ and $m d$ states, followed by a short discussion and interpretation of the experimental material in Sec. IV C. Section V contains a more detailed delineation of some aspects connected with our MCDF calculations.

\section{EXPERIMENTAL METHODS}

Experimental results of this work were obtained at two subsequent runs at the synchrotron radiation facilities BESSY (Berlin, Germany) and Hasylab (Hamburg, Germany). In the following the former are called "low resolution" and the latter "high resolution"' results. For both runs a rotatable vacuum chamber equipped with two time-offlight (TOF) analyzers for collection of the electron spectra was used. The TOF spectrometers are mounted in a plane perpendicular to the propagation axis of the synchrotron radiation, and the whole chamber including the spectrometers can be rotated around this axis. Microchannel plates together with a setup of commercially available timing electronics are used to detect the electrons and store the signals into a multichannel analyzer. Further aspects of this setup have been described elsewhere [21]. The length of the flight path of 679 $\mathrm{mm}$ results in times of flight on the order of $200 \mathrm{~ns}$ for the kinetic energies of interest, provided no retarding potentials are employed. To prevent overlapping of lines in the TOF spectra, our measurements had to be taken while the synchrotron radiation facilities operated with a reduced number of electron bunches. At BESSY a single bunch mode with a 208 ns period was used, while Hasylab operated with a dual bunch mode of $480 \mathrm{~ns}$. Atomic $\mathrm{Mg}$ was produced in a resistively heated oven operating at approximately $500{ }^{\circ} \mathrm{C}$ [22].

We performed our measurements at BESSY at the TGM5 toroidal grating monochromator [23] using photons from the undulator U1. At Hasylab we used the BW3 undulator equipped with an SX-700 plane grating monochromator [24]. The optical design of the U1-TGM5 beamline consists of only two optical elements compared to five at BW3-SX700. Thus, in the VUV photon energy range from 50 to $90 \mathrm{eV}$ the absolute photon flux at the sample is of the same order of magnitude for both beamlines, as we determined by comparison of Ne photoionization count rates at a normalized sample pressure. The photon bandpass was about $80 \mathrm{meV}$ at BESSY sufficient to isolate the first six $\mathrm{Mg} 2 p$ resonances. At Hasylab our aim was to isolate the higher members of the $n s$ from the $m d$ Rydberg series. Therefore, we operated with a bandpass of $35 \mathrm{meV}$, which provided an excellent compromise between intensity and resolution. We extracted both values for the bandpass from scans of the total electron yield covering the $2 p$ resonance series. The natural widths of these resonances are below $1 \mathrm{meV}$ [5] and were neglected in the determination of the bandpass. The values of the degree of linear polarization $p_{1}$ and the tilt angle $\lambda$ of the ellipse of light polarization were determined from the comparison of Ne photoelectron angular distributions with reference measurements. Values of the $\mathrm{Ne} 2 p$ angular distribution were taken from [25]. For the $\mathrm{Ne} \sigma_{2 p} / \sigma_{2 s}$ cross section ratio unpublished measurements of our group were used, as discussed below. We always found $p_{1}>0.95$ and $|\lambda|<3^{\circ}$.

During both runs, we first recorded scans of the total elec- 
tron yield versus photon energy to find the positions of the resonances on the monochromator's energy axis and to find a suitable compromise between the width of the photon bandpass and the count rate. Once the energy positions of the resonances were known, we recorded energy resolved electron spectra at each resonance energy. The chamber was rotated subsequently to three different angles with regard to the electric field vector of the impinging photons. Counting times were between 500 and $1000 \mathrm{~s}$ for each angle. Thus for determination of the angular distributions, spectra under six different analyzer angles were available at BESSY. At Hasylab, only one spectrometer was operational, so the angular distributions were determined from spectra taken under $0^{\circ}$, $-40^{\circ}$, and $-53^{\circ}$ ("quasimagic angle"). For the low resolution measurements, the drift tubes of the TOF spectrometers were kept at ground potential. This resulted in a full width at half maximum (FWHM) of an isolated line which varied from $400 \mathrm{meV}$ to $550 \mathrm{meV}$ in a typical spectrum (note that the resolution of a TOF analyzer varies linearly as a function of kinetic energy). For the high resolution measurements a voltage of $-27.5 \mathrm{~V}$ was used to retard the electrons to further improve the resolution of the spectra. This yielded a FWHM which varied from $130 \mathrm{meV}$ to $310 \mathrm{meV}$ in a typical spectrum.

The aim of the data analysis was to extract values of the branching ratios of the various resonant Auger lines at a given excitation energy, and of the asymmetry parameter $\beta$ which describes their angular distribution. The former requires a comparison of the intensity of all electron lines in one spectrum measured at the quasimagic angle (this assures that the measured intensities are independent of angular distribution effects). The latter requires a comparison of the intensity of one electron line from different spectra taken under different angles. As a result, the calibration procedures to account for systematic errors resulting from, e.g., the dependence of the spectrometer transmission on experimental variables, are different for the two different types of measurements.

The areas of most lines were determined by adding the counts within a region of interest and subtracting a linear background. Net areas were between 500 and 50000 counts for the weakest and most intense features. For some pairs of adjacent lines, the spectra were converted from the time of flight to a kinetic energy scale. Then the intensity of the lines was determined by least- $\chi^{2}$ fitting a profile consisting of two Gaussians to the data.

To calibrate the areas for extraction of the branching ratios the relative transmission function of the analyzer at the quasimagic angle was determined from a set of Ne calibration spectra. Ne gas was admitted into the chamber through the oven nozzle. We define a "relative transmission function" $T_{\text {rel }}\left(E_{\mathrm{kin}}\right)$, which is calculated from the cross-section normalized area ratio of the $\mathrm{Ne} 2 s$ and $2 p$ photoionization main lines:

$$
T_{\text {rel }}\left(E_{\text {kin }}\right):=\frac{A_{2 s}(h \nu)}{A_{2 p}(h \nu)} \frac{\sigma_{2 p}(h \nu)}{\sigma_{2 s}(h \nu)} .
$$

$A_{n l}$ denotes the area of the $n l$ photoionization line. $E_{\text {kin }}=h \nu-E_{b}(2 s)$ is the kinetic energy of the Ne $2 s$ line. At kinetic energies above $100 \mathrm{eV}$, values of $T_{\text {rel }}$ approach 1 as the analyzer transmission gets similar for both lines. Towards lower kinetic energies, the transmission function [Eq. (3)] and the relative transmission function usually rise due to electron optical effects in the TOF analyzers. At kinetic energies below $1-5 \mathrm{eV}$, they rapidly drop to zero. For low kinetic energies, the influence of the transmission function on the divisor $A_{2 p}$ can be recursively corrected starting at a value of $T_{\text {rel }}\left(E_{\mathrm{kin}}\right)$ above $100 \mathrm{eV}$.

The cross-section ratios entering $T_{\text {rel }}$ were taken from unpublished measurements carried out within our group. Their values agree well with the literature [26] above a photon energy of $90 \mathrm{eV}$, while they are smaller by at most $1.3 \%$ of the total photoionization cross section below this energy. In fact, only the slope of this function has an influence on the determination of the branching ratios. Its uncertainty has been taken into consideration in the experimental error bars, but is of minor importance for this work. The photon energies used to record the values of $T_{\text {rel }}$ must be chosen such that the kinetic energy of the $\mathrm{Ne} 2 s$ line spans the region of kinetic energies of interest for the $\mathrm{Mg}$ resonant Auger spectra. In this context it is important to note that the flux curve of the monochromator cancels from the expression for $T_{\text {rel }}$. Branching ratios can be determined directly from a set of resonant Auger areas in a quasimagic angle spectrum divided by $T_{\text {rel }}$ at the pertinent kinetic energies.

Division by $T_{\text {rel }}$ is not suitable to normalize lines of identical kinetic energies which have been recorded at different spectrometer angles. This is because the transmission function might exhibit a dependence on the spectrometer angle which is common to all kinetic energies. One possible reason is the anisotropy of the source volume due to the shape of the synchrotron radiation light beam and the cone of Mg effusing from the oven. A suitable normalization in this case is the transmission function $T$ defined by

$$
T\left(\phi, E_{\text {kin }}\right):=A_{2 s}^{i}(\phi, h \nu) /\left(W_{2 s}(\phi) I^{i}\right),
$$

where $A_{2 s}^{i}$ is the area of the Ne $2 s$ line in the $i$ th spectrum and $W_{2 s}$ is its angular distribution function (4) having a $\beta$ value of 2 . Here $\phi$ describes the angle between the orbit plane of the synchrotron and the spectrometer, and $I^{i}$ is a measure of the photon intensity accrued during accumulation of the $i$ th spectrum.

Before and after recording each set of resonant spectra a spectrum of the Mg $L M M$ normal Auger group at a fixed photon energy above the $2 p$ thresholds was taken. These spectra were used for correcting fluctuations of the oven intensity. In most cases these fluctuations were below 5\%, however the maximum value was as high as $100 \%$. The areas of all spectra bracketing the resonance series were normalized to the photon intensity. For each resonant spectrum the spline interpolation of these values to the moment, where half its acquisition time had passed, was taken as a correction factor. For each resonant Auger line we determined a set of corrected areas by dividing with the appropriate value of the $T$ function and the correction factor for the oven fluctuations. These corrected areas were fitted by the angular distribution function

$$
W(\phi)=\frac{\sigma}{4 \pi}\left[1+\frac{1}{4} \beta\left[1+3 p_{1} \cos 2(\phi-\lambda)\right],\right.
$$


TABLE I. Energy levels of $\mathrm{Mg}$ I in the region of the $2 p$ resonances. The numerical resonance designations follow Whitfield et al. [4]. $E_{\text {opt }}$ is the excitation energy of the level relative to the ground state according to Newsom [5], $\Delta E_{\mathrm{opt}}$ is the energy relative to the first $2 p$-excited level. The assignments are taken from our MCDF calculations. The sum of the weights of all configurations contributing to a level is 1 .

\begin{tabular}{lccccc}
\hline \hline $\begin{array}{l}\text { Res. } \\
\text { No. }\end{array}$ & $\begin{array}{c}E_{\mathrm{opt}}^{\mathrm{a}} \\
(\mathrm{eV})\end{array}$ & $\begin{array}{c}\Delta E_{\mathrm{opt}} \\
(\mathrm{eV})\end{array}$ & $\begin{array}{c}\Delta E_{\mathrm{th}} \\
(\mathrm{eV})\end{array}$ & weight & $\begin{array}{c}\text { Assignment } \\
\text { config. }\end{array}$ \\
\hline 1 & $54.8006(17)$ & & $53.0448^{\mathrm{b}}$ & 0.88 & $2 p_{3 / 2}^{-1} 4 s$ \\
2 & $54.9602(17)$ & 0.160 & $0.120^{\mathrm{c}}$ & 0.91 & $2 p^{-1} 3 s p^{2}$ \\
& & & $0.236^{\mathrm{c}}$ & 0.70 & $2 p^{-1} 3 s 3 p^{2}$ \\
3 & $55.0645(17)$ & 0.264 & 0.273 & 0.89 & $2 p_{1 / 2}^{-1} 4 s$ \\
& & $0.42^{\mathrm{d}}$ & 0.462 & 0.96 & $2 p_{3 / 2}^{-1} 3 s 3 p^{2}$ \\
4 & $55.4916(15)$ & 0.691 & 0.720 & 0.72 & $2 p_{3 / 2}^{-1} 3 d$ \\
5 & $55.6770(15)$ & 0.876 & 0.910 & 0.35 & $2 p_{3 / 2}^{-1} 3 d$ \\
6 & $55.8377(15)$ & 1.037 & 1.086 & 0.42 & $2 p_{1 / 2}^{-1} 3 d$ \\
7 & $56.2802(16)$ & 1.480 & 1.311 & 0.91 & $2 p_{3 / 2}^{-1} 5 s$ \\
$8 \mathrm{a}$ & $56.3421(13)$ & 1.542 & 1.487 & 0.87 & $2 p_{3 / 2}^{-1} 4 d$ \\
$8 \mathrm{~b}^{\mathrm{e}}$ & $56.3660(12)$ & 1.565 & 1.525 & 0.82 & $2 p_{3 / 2}^{-1} 4 d$ \\
9 & $56.5451(13)$ & 1.745 & 1.592 & 0.91 & $2 p_{1 / 2}^{-1} 5 s$ \\
10 & $56.6032(13)$ & 1.803 & 1.770 & 0.86 & $2 p_{1 / 2}^{-1} 4 d$ \\
$11 \mathrm{a}^{\mathrm{e}}$ & $56.7855(13)$ & 1.985 & 2.552 & 1.0 & $2 p_{3 / 2}^{-1} 6 s$ \\
$11 \mathrm{~b}$ & $56.8156(13)$ & 2.015 & & & $2 p_{3 / 2}^{-1} 5 d^{\mathrm{f}}$ \\
12 & $57.0809(16)$ & 2.280 & & & $2 p_{1 / 2}^{-1} 5 d, 2 p_{3 / 2}^{-1} 6 d^{\mathrm{f}}$ \\
\hline \hline
\end{tabular}

${ }^{\mathrm{a} C a l c u l a t e d}$ using $E(\mathrm{eV})=12398.424 / \lambda(\AA)$, CODATA 1987 recommendations.

${ }^{\mathrm{b}}$ Energy relative to the theoretical $\mathrm{Mg}$ ground state.

${ }^{\mathrm{c}}$ The assignment of this resonance is unclear.

${ }^{\mathrm{d}}$ The photographic plate of Newsom [5] shows a faint blackening at this energy, which is not discussed by this author.

${ }^{\mathrm{e}}$ Not observed in this work.

${ }^{\mathrm{f}}$ Assignment by Newsom [5].

where $p_{1}$ is the degree of linear polarization of the light and $\lambda$ the tilt angle of the electric field vector measured from the orbit plane of the synchrotron. This fitting procedure yields two parameters: the anisotropy parameter $\beta$ and a parameter proportional to the total cross section $\sigma$. However, as we are not able to determine our target density in absolute values, we cannot put the result for the cross section on an absolute scale.

\section{THEORY}

To understand our experimental observations on a quantitative level, we have performed multiconfiguration DiracFock (MCDF) calculations of the branching ratios and angular anisotropy parameters of the first seven resonances.

Our calculations are based on the two-step model of resonant Auger decay. The possibility of interference with the direct pathway is always ignored. This seems to us an approximation well justified by the following reasoning.

Most of the excited states reached by $2 p$ photoexcitation in $\mathrm{Mg}$ are well isolated (in this context well isolated means there is negligible overlap between adjacent Rydberg levels, which implies that lifetime interference effects can be ignored). Moreover, in general their decay to a given final state dominates over the population of that state via direct photoionization [4].

Direct $3 s$ photoionization may contribute to the resonant participator channels. In the constant ionic state (CIS) spec- tra of Whitfield et al. [4] the off-resonant intensity of the $[\mathrm{Ne}] 3 s$ final state was between approximately $20 \%$ and less than $1 \%$ of the resonant intensity for the weakest and strongest resonance (nos. 2 and 4, see Table I). This is clearly an upper limit for the direct contributions, as the monochromator bandpass smears out the resonant features. Still, at certain points for the participator channel we note deviations from our theory, which might be due to direct transitions. These cases are discussed below as they occur.

Besides the $3 s^{-1}$ final state only three satellite states are populated outside the resonances at all: $[\mathrm{Ne}] 3 p$ and $[\mathrm{Ne}] 4 s, 3 d$ [4]. If these lines are populated from the $\mathrm{Mg}$ $[\mathrm{Ne}] 3 s^{2}$ ground state, their intensity arises from matrix elements of the form $\langle 3 s \mid \varepsilon s\rangle\langle 3 s\|d\| n l\rangle$ or $\langle 3 s \mid n s\rangle\langle 3 s\|d\| \varepsilon l\rangle$, where $d$ is the dipole operator. Both of these combinations were found to be small in an earlier theoretical study [10]. Another source for nonresonant population of satellite states can be ground state configuration interaction, namely with doubly excited states of the type $[\mathrm{Ne}] 3 p^{2}$. Experimentally the nonresonant intensity of the aforementioned lines is smaller by at least a factor of 10 compared to the [Ne] $3 \mathrm{~s}$ main line [4]. Therefore, for final states different than $[\mathrm{Ne}] 3 s$ we do not expect measurable contributions from direct transitions.

We note that ground state configuration interaction (GSCI) can have an affect on our results for the decay only via the neglected direct transitions. As long as the two-step approximation holds, the ground state will have no influence 
on the nature of the resonantly excited state and consequently neither on its decay parameters. However, GSCI might influence the value of the absolute theoretical excitation energy given in Table I. Our figure was calculated for a single configuration $\left([\mathrm{Ne}] 3 s^{2}\right)$ ground state.

\section{A. Spectator model predictions}

It is instructive to review some predictions for the anisotropy of resonant Auger decay derived from simple considerations. In a two-step model of Auger and resonant Auger decay the anisotropy parameter can be factorized as $\beta=\alpha_{2} \mathcal{A}_{20}[27,28]$. Only the intrinsic parameter $\alpha_{2}$ contains the dynamics of the radiationless decay, while the alignment parameter $\mathcal{A}_{20}$ is determined solely by the excitation process. The latter is proportional to the intermediate state's electric quadrupole moment. For excitation of a system whose total momentum in the ground state is $J=0$ by perfectly linearly polarized light, the alignment is a fixed value, $\mathcal{A}_{20}=-\sqrt{2}$. This results entirely from dipole selection rules [29]. We define $\mathcal{A}_{20}$ with respect to a coordinate system whose $z$ axis extends along the direction of the electric-field vector of the incoming radiation.

Therefore, only models for the intrinsic parameter $\alpha_{2}$ need to be developed. By dipole selection rules excitation may take place from a $J=0$ ground state only to a $J=1$ intermediate state. In this case $\alpha_{2}$ has the following form valid for spectator or participator decay $[27,28]$ :

$$
\begin{gathered}
\alpha_{2}=\frac{1}{W_{0}}(-1)^{J_{f}+1 / 2} \sqrt{3} \sum_{l l^{\prime} j j^{\prime}} \hat{l} \hat{l}^{\prime} \hat{j} \hat{j}^{\prime}\left\langle l 0, l^{\prime} 0 \mid 20\right\rangle\left\{\begin{array}{ccc}
1 & 1 & 2 \\
j & j^{\prime} & J_{f}
\end{array}\right\} \\
\times\left\{\begin{array}{ccc}
j & j^{\prime} & 2 \\
l^{\prime} & l & \frac{1}{2}
\end{array}\right\}\left\langle J_{f}, j: 1\|V\| 1\right\rangle\left\langle J_{f}, j^{\prime}: 1\|V\| 1\right\rangle^{*} \\
W_{0}=\sum_{j}\left|\left\langle J_{f}, j: 1\|V\| 1\right\rangle\right|^{2}
\end{gathered}
$$

where $J_{f}$ is the final total momentum of the ion after decay, $l, l^{\prime}$ and $j, j^{\prime}$ refer to the orbital and total angular momentum of the continuum electron, and $\hat{a} \equiv \sqrt{2 a+1}$. The quantity $\left\langle l 0, l^{\prime} 0 \mid 20\right\rangle$ is a Clebsch-Gordan coefficient, while the factors in curly brackets are $6 j$ symbols, and the radiationless transition matrix element is represented by the quantity in angular brackets.

For the spectator decay of excited $\operatorname{Mg} 2 p^{-1} n s(n>3)$ states, some results can be drawn from simple considerations. We use the frozen-core approximation for the singleparticle wave functions and employ $j j$ coupling. In this case, the wave function of the spectator electron can be removed from the many-particle Coulomb matrix element by a recoupling procedure [30-32]:

$$
\begin{aligned}
& \left\langle\left(\left(2 p^{6}\right) J_{c},(n s) j_{1}\right) J_{f},(\varepsilon p) j: 1\|V\|\left(2 p^{-1}\right) j_{0},\left(3 s^{2}\right) 0,(n s) j_{1}: 1\right\rangle=(-1)^{J_{f}+j_{1}+j+j_{0}} \sqrt{3} \hat{J}_{f}\left\{\begin{array}{ccc}
j & 1 & J_{f} \\
j_{1} & J_{c} & j_{0}
\end{array}\right\} \\
& \quad \times\left\langle\left(2 p^{6}\right) J_{c},(\varepsilon p) j: j_{0}\|V\|\left(2 p^{-1}\right) j_{0},\left(3 s^{2}\right) 0: j_{0}\right\rangle .
\end{aligned}
$$

Here $J_{c}$ denotes the core total angular momentum, $j_{0}$ the angular momentum of the initial vacancy, and $j_{1}$ the angular momentum of the spectator electron. In our case we have $j_{0}=1 / 2$ or $3 / 2$ and $J_{c}=0$. Consequently, we get $j_{0}=j$. That is, a $2 p_{3 / 2}^{-1}\left(2 p_{1 / 2}^{-1}\right)$ vacancy state can only decay via emission of an $\varepsilon p_{3 / 2}\left(\varepsilon p_{1 / 2}\right)$ electron. These restrictions originate from the two $3 s$ electrons which are directly involved in the decay. As they are equivalent, their angular momenta must couple to 0 .

When the $\alpha_{2}$ parameter involves the emission of a single partial wave, then it generally is independent of the Auger decay matrix elements and can be calculated analytically [27]. Input parameters in this case are the orbital and total angular momentum of the continuum electron and the total angular momenta of the intermediate and final state. In our case, we have decay of a $J=1$ intermediate state to a [Ne]ns final state with $J_{f}=1 / 2$. For excitation of a $2 p_{1 / 2}$ electron we must set $j^{\prime}=j=1 / 2$ in Eq. (5), which causes the first $6 j$ symbol to vanish and consequently $\alpha_{2}$, leading to $\beta=-\sqrt{2} \alpha_{2}=0$. For the case of $2 p_{3 / 2}$ excitation, $j^{\prime}=j=3 / 2$ and Eq. (5) reduces to $\alpha_{2}=-1 / \sqrt{2}$, which leads to $\beta=1$. Moreover, any deviation of the $\beta$ parameters in the spectator decay of $2 p \rightarrow n s$ excitations from these values must result from a mixing of $2 p^{-1}$ vacancy states with opposite spin-orbit coupling.

We would like to remark that these considerations do not depend on the principal quantum number $n$ of the primary excitation. That is, they will apply to the spectator decays of all $2 p \rightarrow n s$ excitations. Intuitively one would expect the influence of the outer electron on the core vacancies to become smaller for higher $n$. This will entail a smaller mixing between $2 p_{1 / 2}^{-1}$ and $2 p_{3 / 2}^{-1}$ and thus $\beta$ values that adhere better to the spectator model prediction.

For transitions involving shakeup of the spectator electron in a first approximation only an overlap factor accounting for the nonorthogonality of the initial and final outer electron orbitals is multiplied with the transition amplitude [33]. This approximation has been termed "shake theory." As long as it holds, the $\beta$ parameters of normal and shake-modified spectator decay are identical [31]. The influence of relaxation on the anisotropy has only been studied for normal Auger decay beyond this approximation. It was found to be small [34].

To separate the effects of angular momentum coupling from changes of the radiationless dynamics induced by the 
outer electron, Hergenhahn et al. developed the "strict spectator model"' [30,35]. Values for $\beta$ yielded by this model are identical to the numbers calculated above. However, this model assumes that the core electron's wave functions are not changed by the presence of the spectator electron. The present derivation for the $\mathrm{Mg}$ case holds even if one neglects this condition and it is, therefore, more general.

For the participator decays of the $2 p \rightarrow n s$ excitations, the two-particle Coulomb matrix element of Eq. (6) contains two nonequivalent electrons on its right-hand side, namely $3 \mathrm{~s}$ and $n s$. These may couple their angular momenta to values different from 0 . Therefore, in the case of the participator decay, $\varepsilon p_{1 / 2}$ and $\varepsilon p_{3 / 2}$ partial waves are always possible. Suppose that the phase difference between them is negligible. Then according to Eq. (5) and recalling $\mathcal{A}_{20}=-\sqrt{2}$, $\beta$ reduces to

$$
\beta=\frac{2 \sqrt{2} r+1}{1+r^{2}}
$$

where $\quad r \equiv\left\langle J_{f}=\frac{1}{2}, j=\frac{1}{2}: 1|V| 1\right\rangle /\left\langle J_{f}=\frac{1}{2}, j=\frac{3}{2}: 1|V| 1\right\rangle$. This expression is valid for participator decay as well as for spectator decay in the presence of the mixing of vacancy states. For pure $\varepsilon p_{3 / 2}$ decay, $r=0$ and thus $\beta=1$, while for predominance of $\varepsilon p_{1 / 2}$ emission, $\beta$ tends asymptotically towards 0 . Between these two extremes, arbitrary values ranging from -1 to 2 are reached. A remarkable feature of this expression is the steep slope around $r=0$. This implies that the value of $\beta$ for spectator decay is a sensitive indicator of the degree of mixing of the $2 p_{3 / 2}^{-1} n s$ vacancy state. For participator decay, results strongly deviating from $\beta=2$, the value for direct $3 s$ photoionization, may be expected.

\section{B. Numerical calculations}

The technical details of our (MCDF) calculations were discussed partly in [3]. For ease of reference we repeat here all essential points.

We used the MCDF code of Grant et al. [36] to construct all bound state wave functions, while the continuum wave function and the transition rates were calculated using the multichannel MCDF code described in Ref. [37] neglecting, however, continuum-channel interaction. The continuum orbitals were obtained by solving the Dirac equation, including the exchange interaction, in the frozen-core field of the finalionic state. Due to the nonlocality of the exchange potential, the use of an iterative procedure was necessary to calculate the continuum orbitals as well. Orthogonality to bound-state orbitals of the same symmetry was enforced by Lagrange multipliers. For the calculation of the transition rates we used orbitals optimized for the intermediate state throughout.

While no notable configuration interaction $(\mathrm{CI})$ occurs in the final state, the effect of CI in the intermediate state was decisive in the understanding of the experimental spectra. This deviates from findings for the resonant Auger decay of noble gases [38]. To explore the effect of CI on the angular anisotropy of the resonant Auger decay, we used basis sets constructed from one (1C), two (2C), and fourteen (14C) nonrelativistic configurations. Each basis set consisted of all $j j$-coupled states with $J=1$ that could be constructed from the nonrelativistic configurations where (i) $1 \mathrm{C}$ consists of the
$2 p^{-1} 4 s$ configuration for resonances 1 and 3 , and a $2 p^{-1} 3 d$ configuration for resonances $4-6$; (ii) $2 \mathrm{C}$ includes in addition to $1 \mathrm{C}$ the $2 p^{-1}\left(3 p^{2}\right)_{J=0} 4 s$ configuration for resonances 1 and 3 leading to 6 configurations in $j j$ coupling, and a $2 p^{-1} 3 s 3 p^{2}$ configuration for the remaining resonances leading to $14 \mathrm{jj}$-coupled configurations; (iii) $14 \mathrm{C}$ corresponds to a $2 p^{-1}\left\{3 s^{2} n s(n=4-8)+3 s^{2} 3,4 d+3 s 3 p^{2}\right.$ $\left.+3 s 3 d^{2}+3 s 4 s^{2}+\left(3 p^{2}\right)_{J=0} 4,5 s+\left(3 p^{2}\right)_{J=0} 3,4 d\right\}$ intermediate state, leading to $70 j j$-coupled configurations.

The results obtained using the $14 \mathrm{C}$ basis set are displayed in the tables of the following section. Further effects of the basis size on the calculated results are discussed in Sec. V.

In Ref. [3] we took relaxation partly into account by using final state mixing coefficients that were obtained in a separate SCF calculation of the final state wave functions. We do not adhere to this approach in this work. That is, the final state mixing coefficients we use here were obtained by solving the CI equation for the final state in a basis space spanned by the initial state orbitals [36]. This leads to some mixing of final states of the type $[\mathrm{Ne}] n s$, e.g., $[\mathrm{Ne}] 4 s$ with $[\mathrm{Ne}] 5 s$. While these intereferences do not occur in an SCF calculation of the final state, they might be legitimate as a result of the CI calculation. Some admixture of the initial state $5 s$ with the initial state $4 s$ orbital might lead to an improvement of the $4 s$ orbital which more accurately reflects the true final state $4 s$ orbital. However, the energies yielded by a CI calculation of the final states are not improved by the mixing. Thus, we can give no clear preference to either one of the approximations-separately optimized or by a CI calculation-for the mixing coefficients. The influence on the results is in most cases small.

\section{EXPERIMENTAL RESULTS}

Table I shows the energies and assignments of the first $\operatorname{Mg} 2 p$ excited levels below the threshold for $2 p$ ionization. The strongest feature in the absorption and electron yield spectra is the $2 p \rightarrow 3 d$ excitation (resonance 4 , where we have adopted the numerical designations as given in Ref. [4]). However, all of the $3 d$ resonances (4-6) strongly interact with double excitations of the type $2 p^{-1} 3 s 3 p^{2}$, as already supposed in [7]. We will further discuss this point together with the branching ratios for the $d$-like resonances. The optical energies from [5] are consistent with our total electron yield scans within our monochromator stepwidth $(10-20 \mathrm{meV})$. The calculated energy splittings for the highest excited states might not be very reliable due to the influence of even higher Rydberg excitations not included in the basis set.

Levels 8 and 11 are seen as double lines in the absorption spectrum of Newsom [5]. In order to verify this observation, we performed an additional high resolution total electron yield scan with an estimated bandpass of $10 \mathrm{meV}$. Although this is lower than the $15 \mathrm{meV}$ bandpass of Ref. [5], we observed no evidence of a double structure for any of these lines. Later absorption work reproduced a double structure for level 11, but not for level 8 [7]. Our high resolution electron yield scans had a background from thermal electrons comparable to the signal height. Therefore, we cannot rule out the existence of a very weak feature, which was seen in absorption. Energy resolved electron spectra were taken 
TABLE II. Branching ratios into the different final ionic states for resonances with dominant contributions from $2 p^{-1} n s$ configurations. The designations of the $[\mathrm{Ne}] n l$ final states are abbreviated by $n l$. Denoted in parentheses is the dominant configuration of the intermediate state. Expt. (a) and (b) are the low and high resolution results of this work. For comparison results of Whitfield et al. [4] and theoretical values (Theor.) taken from the $14 \mathrm{C}$ calculation of this work are given. The fine structure for decays to final states of $p$ and $d$ symmetry is not resolved. Binding energies $E_{b}$ are taken from the tables of Moore [42] and extended to high $n$ by a quantum defect calculation [4].

\begin{tabular}{|c|c|c|c|c|c|c|c|c|c|}
\hline \multirow[b]{2}{*}{$n l$} & \multirow[b]{2}{*}{$E_{b}(\mathrm{eV})$} & \multicolumn{3}{|c|}{ Res. $1\left(2 p_{3 / 2}^{-1} 4 s\right)$} & \multicolumn{2}{|c|}{ Res. $7\left(2 p_{3 / 2}^{-1} 5 s\right)$} & \multicolumn{3}{|c|}{ Res. $11\left(2 p_{3 / 2}^{-1} 6 s, 5 d\right)$} \\
\hline & & Expt. (a) & Expt. [4] & Theor. & Expt. (b) & Theor. & Expt. (a) & Expt. (b) & Expt. [4] \\
\hline $3 s$ & 7.65 & $15.6(5)$ & $12.6(4)$ & 10.5 & $9.5(5)$ & 2.6 & $9.3(5)$ & $<1$ & $4.1(3)^{\mathrm{a}}$ \\
\hline $3 p$ & 12.07 & $4.2(5)$ & $3.7(2)$ & 2.6 & 1.1(2) & 1.0 & $2.8(5)$ & 2.4(4) & $2.4(3)^{\mathrm{a}}$ \\
\hline $4 s^{\mathrm{b}}$ & 16.30 & 64.1(30) & 67(3) & 80.8 & $2.7(3)$ & 4.8 & 0 & $<1$ & $0^{\mathrm{a}}$ \\
\hline $4 p$ & 17.64 & $7.8(5)$ & 7.6(2) & $0^{c}$ & $<1$ & $0^{c}$ & 0 & 0 & $0^{\mathrm{a}}$ \\
\hline $5 s^{\mathrm{b}}$ & 19.15 & $7.7(5)$ & $7.9(2)$ & 6.1 & $47.9(5)$ & 79.3 & $9.0(5)$ & $9.3(4)$ & 7.4(5) \\
\hline $5 p$ & 19.73 & & $0.5(2)$ & $0^{c}$ & 7.6(3) & $0^{c}$ & 0 & $0.6(3)$ & $0.9(1)$ \\
\hline $6 s^{\mathrm{b}}$ & 20.44 & $<1^{\mathrm{d}}$ & $0.9(1)$ & 0 & $28.5(4)$ & 12.1 & $20.0(10)^{\mathrm{e}}$ & 18.4(4) & 17.7(7) \\
\hline $6 p$ & 20.74 & & & & $1.9(3)$ & $0^{c}$ & & $5.9(3)$ & $6.5(4)$ \\
\hline $7 s, 6 d$ & 21.14 & & & & & & $59.0(20)^{\mathrm{f}}$ & $51.9(8)$ & $52(2)$ \\
\hline $7 p$ & 21.30 & & & & & & & $6.8(8)$ & $6.1(8)$ \\
\hline $8 s, 7 d$ & 21.55 & & & & & & & $3.5(4)$ & $3.2(4)$ \\
\hline
\end{tabular}

\begin{tabular}{|c|c|c|c|c|c|c|c|}
\hline \multirow[b]{2}{*}{$n l$} & \multirow[b]{2}{*}{$E_{b}(\mathrm{eV})$} & \multicolumn{2}{|c|}{ Res. $3\left(2 p_{1 / 2}^{-1} 4 s\right)$} & \multicolumn{2}{|c|}{ Res. $9\left(2 p_{1 / 2}^{-1} 5 s\right)$} & \multicolumn{2}{|c|}{ Res. $12\left(2 p_{1 / 2}^{-1} 5 d, 2 p_{3 / 2}^{-1} 6 d\right)^{\mathrm{g}}$} \\
\hline & & Expt. (a) & Expt. [4] & Theor. & Expt. (b) & Expt. (a) & Expt. [4] \\
\hline $3 s$ & 7.65 & $12.4(5)$ & $9.2(4)^{\mathrm{a}}$ & 27.8 & $2.6(4)$ & $1.5(4)$ & $0^{\mathrm{a}}$ \\
\hline $3 p$ & 12.07 & $6.7(5)$ & $6.8(3)$ & 0.9 & $5.1(3)$ & $2.2(4)$ & $2.2(2)^{\mathrm{a}}$ \\
\hline $4 s^{\mathrm{b}}$ & 16.30 & $63.4(30)$ & $66(3)$ & 66.0 & $2.6(3)$ & $1.1(4)$ & $0^{\mathrm{a}}$ \\
\hline $4 p$ & 17.64 & $8.3(5)$ & $8.3(4)$ & $0^{\mathrm{c}}$ & $<1$ & $0.7(3)$ & $0^{\mathrm{a}}$ \\
\hline $5 s^{\mathrm{b}}$ & 19.15 & $8.3(5)$ & $8.3(4)$ & 5.3 & $50.7(5)$ & $6.7(4)$ & $6.3(4)$ \\
\hline $5 p$ & 19.73 & & & $0^{c}$ & $8.4(3)$ & $0.6(3)$ & $0.6(1)$ \\
\hline $6 s, 5 d$ & 20.45 & $<1^{\mathrm{d}}$ & $1.3(7)^{\mathrm{d}}$ & 0 & $27.7(4)$ & $14.8(3)$ & $15.3(6)$ \\
\hline $6 p$ & 20.74 & & & & 2.1(3) & $4.6(2)$ & $4.8(2)$ \\
\hline $7 s, 6 d$ & 21.14 & & & & $0.5(2)$ & $34.5(5)$ & $40(2)$ \\
\hline $7 p$ & 21.30 & & & & & $5.5(5)$ & $\mathrm{h}$ \\
\hline $8 s, 7 d$ & 21.55 & & & & & $16.1(5)$ & $25(1)^{\mathrm{i}}$ \\
\hline $8 p$ & 21.67 & & & & & $6.2(5)$ & $\mathrm{h}$ \\
\hline $9 s, 8 d$ & 21.82 & & & & & $5.6(5)$ & $5.5(3)$ \\
\hline
\end{tabular}

${ }^{\mathrm{a}}$ Contributions of direct transitions has been removed.

${ }^{\mathrm{b}}$ Contribution of $(n-1) d$ possible for Res. 11 and 12.

${ }^{\mathrm{c}}$ Not included in calculation.

${ }^{\mathrm{d}}$ Sum of $5 p$ and $6 s$.

${ }^{\text {e }}$ Sum of $6 s, 5 d$, and $6 p$.

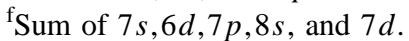

${ }^{\mathrm{g}}$ Contribution of $2 p_{1 / 2}^{-1} 6 s$ and $2 p_{3 / 2}^{-1} 7 s$ possible in our opinion, see text.

${ }^{\mathrm{h}}$ State was established, but could not be resolved.

${ }^{\mathrm{i}} \mathrm{Sum}$ of $8 s, 7 d$, and $8 p$.

with excitation energies of levels $8 \mathrm{a}$ and $11 \mathrm{~b}$ according to Table I.

Within this work we made no attempt to subtract the contributions of direct transitions from the resonant intensity as was carried out in Ref. [4]. The bandpass of the exciting radiation in [4] was $0.30 \AA$ or $90 \mathrm{meV}$ at 60 $\mathrm{eV}$ photon energy. In this work the low- and high-resolution measurements were taken with a bandpass of approximately $80 \mathrm{meV}$ and $35 \mathrm{meV}$, respectively. The bandpass enters the experimental results via convolution with the Fano profile of the partial cross section or the angular distribution parameter
[39] along the resonance. This smears out the resonance properties more the wider the bandpass gets. We therefore expect the $[\mathrm{Ne}] 3 s$ states to be considerably less affected in this work than in [4]. As argued earlier, we do not expect an influence from direct transitions to other final states.

\section{A. Results for $n s$ excitations}

The branching ratios for the decays of the $2 p \rightarrow n s$ excitations are given in Table II. Spectra taken under the quasimagic angle are displayed in Fig. 1. For the lowest 

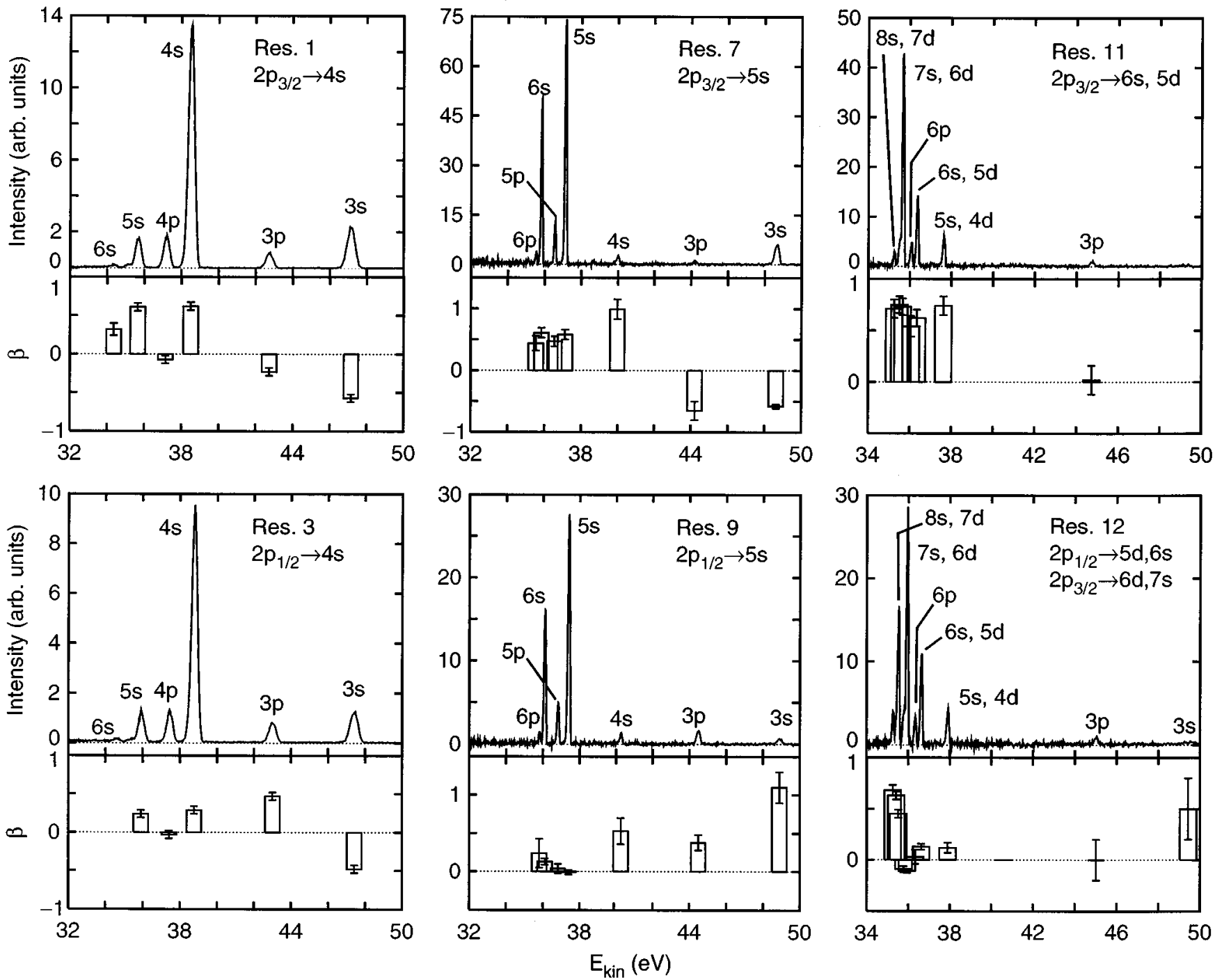

FIG. 1. Electron spectra and the $\beta$ parameter recorded on the $\mathrm{Mg} 2 p \rightarrow n s$ resonances. The classification of resonance 12 is discussed in the text. Spectra for resonances 1 and 3 are taken from the low-resolution experiment. The intensity values displayed are normalized by oven intensity, number of photons accumulated, and relative transmission $T_{\text {rel }}$ (see text). Therefore, intensities of spectra stemming from the same series of measurements can be compared to each other. (1 can be compared to 3, the remaining spectra can be compared among each other, but not with 1 or 3 .) The width of the histogram bars used to indicate the $\beta$ parameter is chosen arbitrarily.

resonances 1 and $3\left(2 p_{3 / 2} \rightarrow 4 s, 2 p_{1 / 2} \rightarrow 4 s\right)$ pure spectator decay to the $[\mathrm{Ne}] 4 s$ final state dominates. It attracts about $2 / 3$ of the total decay intensity. Among the other channels participator decay to the $[\mathrm{Ne}] 3 \mathrm{~s}$ final ionic state is most important. These results are in excellent agreement with the earlier work of Whitfield et al. [4], with the exception of the participator decay at some resonances. Our calculations also predict a dominance of spectator decay. However, the branching ratios for spectator decay tend to come out too high, at the expense of $[\mathrm{Ne}] 3 p$ and $[\mathrm{Ne}] 4 p$ satellite states. The latter were not included in our calculation.

Comparing the results for the $2 p \rightarrow 5 s$ excitations (resonances 7 and 9) to the results for the $2 p \rightarrow 4 s$ excitations, we find the probability for shakeup to be considerably stronger. The growing importance of shakeup decay towards higher $2 p \rightarrow n s$ excitations was already observed earlier $[4,10]$. However, due to limited monochromator resolution this trend could not be experimentally verified for higher lying resonances of the same symmetry.

Values for the anisotropy parameter $\beta$ are given in Table III. The experimental values are also indicated by the bar diagrams in Fig. 1. A first inspection of the experimental $\beta$ values for the $2 p \rightarrow 4 s$ resonances shows marked deviations from predictions of our simple model delineated in Sec. III A. While this model would predict $\beta=1(\beta=0)$ for the spectator decay of the $2 p_{3 / 2}^{-1} 4 s\left(2 p_{1 / 2}^{-1} 4 s\right)$ excited state, the experiment gives values of $0.62(5)[0.29(5)]$. These deviations must originate from a mixing of the $2 p_{3 / 2}^{-1}$ and $2 p_{1 / 2}^{-1}$ hole states. As we will show in Sec. V, the interaction with other (nonrelativistic) configurations sensitively influences this mixing ratio.

Inspection of the results for the $2 p_{3 / 2} \rightarrow 5 s$ excitation indicates little difference in the decay dynamics as compared to the $2 p_{3 / 2} \rightarrow 4 s$ excitation. In contrast to this, however, we 
TABLE III. Asymmetry parameter $\beta$ of the resonant Auger lines following $2 p_{3 / 2} \rightarrow n s$ (upper panel) and $2 p_{1 / 2} \rightarrow n s$ (lower panel) excitation, respectively. Results for resonances 1 and 3 have been given in Ref. [3].

\begin{tabular}{|c|c|c|c|c|c|c|}
\hline \multirow[b]{2}{*}{$n l$} & \multicolumn{2}{|c|}{ Res. $1\left(2 p_{3 / 2}^{-1} 4 s\right)$} & \multicolumn{2}{|c|}{ Res. $7\left(2 p_{3 / 2}^{-1} 5 s\right)$} & \multicolumn{2}{|c|}{ Res. $11\left(2 p_{3 / 2}^{-1} 6 s, 5 d\right)$} \\
\hline & Expt. (a) & Theor. & Expt. (b) & Theor. & Expt. (a) & Expt. (b) \\
\hline $3 s$ & $-0.57(5)$ & -0.23 & $-0.58(3)$ & -0.98 & $1.75(5)$ & a \\
\hline $3 p$ & $-0.23(5)$ & 0.67 & $-0.65(15)$ & 0.36 & $0.28(5)$ & $0.02(14)$ \\
\hline $4 s$ & $0.62(5)$ & 0.82 & $0.99(16)$ & 1.28 & & \\
\hline $4 p$ & $-0.07(5)$ & c & & & & \\
\hline $5 s^{\mathrm{b}}$ & $0.61(5)$ & 0.77 & $0.58(8)$ & 0.92 & $0.96(5)$ & $0.74(10)$ \\
\hline $5 p$ & & & $0.47(8)$ & c & & \\
\hline $6 s^{\mathrm{b}}$ & & & $0.61(8)$ & 0.91 & $0.75(5)^{\mathrm{d}}$ & $0.62(8)$ \\
\hline $6 p$ & & & $0.44(12)$ & c & & $0.54(10)$ \\
\hline $7 s, 6 d$ & & & & & $0.84(5)^{\mathrm{e}}$ & $0.73(9)$ \\
\hline $7 p$ & & & & & & $0.75(9)$ \\
\hline $8 s, 7 d$ & & & & & & $0.71(9)$ \\
\hline
\end{tabular}

\begin{tabular}{|c|c|c|c|c|}
\hline \multirow[b]{2}{*}{$n l$} & \multicolumn{2}{|c|}{ Res. $3\left(2 p_{1 / 2}^{-1} 4 s\right)$} & \multirow{2}{*}{$\frac{\text { Res. } 9\left(2 p_{1 / 2}^{-1} 5 s\right)}{\text { Expt. (b) }}$} & \multirow{2}{*}{$\frac{\text { Res. } 12\left(2 p_{1 / 2}^{-1} 6 s, 2 p_{3 / 2}^{-1} 6 d\right)}{\text { Expt. (b) }}$} \\
\hline & Expt. (a) & Theor. & & \\
\hline $3 s$ & $-0.48(5)$ & -0.78 & 1.1(2) & $0.5(3)$ \\
\hline $3 p$ & $0.47(5)$ & 0.17 & $0.38(10)$ & $0.0(2)$ \\
\hline $4 s^{\mathrm{b}}$ & $0.29(5)$ & 0.34 & $0.53(17)$ & $0.0(4)$ \\
\hline $4 p$ & $-0.03(5)$ & c & & \\
\hline $5 s, 4 d$ & $0.24(5)$ & 0.29 & $-0.02(3)$ & $0.12(5)$ \\
\hline $5 p$ & & & $0.04(6)$ & \\
\hline $6 s, 5 d$ & & & $0.13(4)$ & $0.13(4)$ \\
\hline $6 p$ & & & $0.24(19)$ & $0.03(7)$ \\
\hline $7 s, 6 d$ & & & & $-0.11(4)$ \\
\hline $7 p$ & & & & $-0.09(4)$ \\
\hline $8 s, 7 d$ & & & & $0.45(4)$ \\
\hline $8 p$ & & & & $0.63(4)$ \\
\hline $9 s, 8 d$ & & & & $0.68(5)$ \\
\hline
\end{tabular}

${ }^{\mathrm{a}}$ State has vanishing intensity.

${ }^{\mathrm{b}}$ Contribution of $(n-1) d$ possible for Res. 11 and 12 .

${ }^{\mathrm{c}}$ Not included in calculation.

${ }^{\mathrm{d}}$ Sum of $6 s, 5 d$, and $6 p$. ${ }^{\mathrm{e}}$ Sum of $7 s, 6 d, 7 p, 8 s$, and $7 d$.

observe a significant difference between the decay from the $2 p_{1 / 2} \rightarrow 5 s$ excitation and the $2 p_{1 / 2} \rightarrow 4 s$ excitation. For the former we find $\beta=-0.01(3)$ for the $[\mathrm{Ne}] 5 s$ line, which comes close to the spectator model prediction of 0 .

For shakeup decay of the excited $n s$ electron to the nexthigher value of the principal quantum number, that is decay to a $[\mathrm{Ne}] 5 s$ final ionic state on resonances 1 and 3 and to a [Ne]6s final ionic state for 7 and 9, respectively, we find an angular anisotropy similar to the pure spectator decay. Only resonance 9 , where $\beta$ is $0.13(4)$ for the [Ne] $6 s$ line, does not fully confirm this trend.

The participator decay to the $[\mathrm{Ne}] 3 \mathrm{~s}$ final ionic state shows some strongly negative $\beta$ values for the $4 s, 5 s$ excitations. This observation, as noted in Ref. [3], is in sharp contrast to the participator behavior in the rare gases [40] and other alkaline earth elements where strongly positive $\beta$ values have been observed $[18,19]$. The $\beta$ value for direct $3 s$ photoionization would of course be 2 . This indicates that the strongly negative $\beta$ values are a direct consequence of the dynamics of the radiationless decay.
Our theory shows satisfactory agreement for the dominant decay modes of the lowest resonances. The return towards the spectator model predicted for resonance 7 was not verified experimentally. The angular anisotropy of the decays to $[\mathrm{Ne}] n p$ satellite states could not be reproduced theoretically. Final states having an outer electron of $p$-symmetry can only be reached via configuration interaction (CI). Our asumption is, for the moment, that this poses a CI problem still too complicated for the methods in use. In our earlier paper [3], we performed an incoherent average of the participator $\beta$ value with the appropriately weighted $\beta$ value of 2 for the direct photoionization channel on resonance 3 . The weighting factor was obtained from the CIS scans in Ref. [4] and led to $\beta=0.49$. While this result agrees well with experiment, it is probably fortuitous. Our procedure of incoherent averaging neglects the interference between the direct and indirect pathways. Even besides that, weighting factors taken from a CIS scan with bandpass comparable to the $\beta$ measurement are not appropriate, as they already contain the "smearing out" of resonant features, including the $\beta$ param- 
eter, that we wanted to approximate by performing the average.

Resonance 11 might be an excitation to a $2 p_{3 / 2}^{-1} 6 s$ state or to a $2 p_{3 / 2}^{-1} 5 d$ state or both. These designations claimed in several works all refer to a quantum-defect analysis made by Newsom [5]. Newsom assigns line no. 11a, unobserved in this work, as an excitation to a $6 s$ orbital. Our resonance 11 should then be the $5 d$ excitation. However, Whitfield et al. [4] assign their (single) resonance 11 to a $2 p_{3 / 2}^{-1} 6 s$ state without considering any $5 d$ contribution.

The observed branching ratios are also compatible with the assumption of excitation to a $6 s$ orbital. Decay to final states with binding energies around 20.45 and $21.14 \mathrm{eV}$ dominates. If the excited electron is in a $6 s$ orbital, these resonant Auger electrons would correspond to pure spectator decay and shakeup to the $[\mathrm{Ne}] 7 \mathrm{~s}$ final ionic state. In this case, shakeup of the outer electron would be more likely than the pure spectator decay. This behavior was examined in detail for the Mg case in Ref. [10]; see also Ref. [11]. On resonance 12 the branching ratios have a similar pattern.

The angular anisotropies for resonances 11 show deviations between the low- and high-resolution results. So far we are not able to explain these findings completely. The most plausible assumption seems to be the existence of two overlapping intermediate states at the position of this resonance. Due to the difference in bandpass between the two experiments they could be excited with different weights. Variations caused by the excitation process are immediately apparent for the branching ratios of the participator decay to the $[\mathrm{Ne}] 3 \mathrm{~s}$ final ionic state in the three experiments (this work and Ref. [4]).

On resonance 11, the differences in the angular anisotropy depending on the influence of the outer electron in the $[\mathrm{Ne}] n l$ final state have disappeared. All lines (except the weak transition to $[\mathrm{Ne}] 3 p$ ) have similar $\beta$ values. One could interpret this as a decrease in the importance of dynamical effects-i.e., variations of the radial integrals-while the influence of structural effects-i.e., the mixing ratio between different couplings of the $2 p^{-1}$ vacancy state-does not change. However, for the moment this interpretation is only tentative.

On resonance 12 one group of lines shows an isotropic decay pattern, the other group is positive with similar $\beta$ values. Newsom [5] classified this resonance as a $2 p_{3 / 2} \rightarrow 6 d$ and $2 p_{1 / 2} \rightarrow 5 d$ excitation. In analogy to results for the lower $m d$ excitations (Sec. IV B), one would not expect the decay to highly excited final states to have a positive $\beta$. Moreover, the participator final state $[\mathrm{Ne}] 3 \mathrm{~s}$ should be strongly populated. Assuming the classification of resonance 12 by Newsom [5] is correct, then we observe a qualitative change of the branching ratios and angular anisotropy parameters dependent on the principal quantum number for this resonance. One could take this interpretation even further by assigning the isotropic lines to the decay of the $2 p_{1 / 2}^{-1} 5 d$ excited state, and the positive $\beta$ lines to $2 p_{3 / 2}^{-1} 6 d$.

However, at our present state of knowledge this interpretation is still tentative. To validate it, one has to exclude the possibility of alternative classifications of this resonance. Energetically nearby could be the $2 p_{1 / 2} \rightarrow 6 s$ and $2 p_{3 / 2} \rightarrow 7 s$ excitations. The former is unobserved according to the quan- tum defect analysis by Newsom [5], although this author assigns other resonances to even higher $2 p_{1 / 2} \rightarrow n s$ excitations. The $2 p_{3 / 2} \rightarrow 7 s$ excitation is neither assigned, nor is its absence discussed in any previous absorbtion study [5-7]. In our opinion, these excitations cannot be excluded, and one or both may play a role in the formation of resonance 12 .

\section{B. Results for $m d$ excitations}

Table IV gives the branching ratios on all resonances established as a $2 p \rightarrow m d$ excitation and on resonances where double excitations to $2 p^{-1} 3 s 3 p^{2}$ play a role. The pertinent spectra, including $\beta$ values of the various resonant Auger lines, are displayed in Fig. 2. Table V shows the weights of the most important configurations in the eigenvector decompositions of resonances 4-6. Only the intermediate state of resonance 4 can be assigned to a single $j$ value of the $2 p$ hole, namely $2 p_{3 / 2}^{-1}$. For resonances 5 and 6 both spin-orbit components of the hole state are mixed to form the intermediate state. In our interpretation, this is a peculiarity resulting from the sensitivity of the unfilled $\mathrm{Mg} 3 d$ shell to interaction with other configurations. Resonances $8 \mathrm{a}$ and 10 are assigned to the $2 p \rightarrow 4 d$ excitations. The fine-structure splitting within the $2 p_{3 / 2}^{-1} 4 d(J=1)$ intermediate state cannot be observed. Theoretical values displayed for resonance 8 are calculated for the fine-structure component being lower in energy, because we believe the higher component is not observed in our emission experiment. Resonance 2 consists of an excitation mainly to $2 p^{-1} 3 s 3 p^{2}$.

The dominant decay mode of all $3 d$ excitations (4-6) is the participator decay to the $[\mathrm{Ne}] 3 s$ final ionic state. This is in marked contrast to the decay of all $s$-like excitations. In fact, the first observations of similar decay patterns on the $\mathrm{Ca}$ $2 p \rightarrow 3 d$ resonances were reported as "breakdown of the spectator model" [14]. The second-most important final states, at least for resonances 5 and 6 , are satellite states of the $[\mathrm{Ne}] 3 p$ type. A comparison with Table $\mathrm{V}$ shows that the population of these final states increases with the admixture of $[\mathrm{Ne}] 3 s 3 p^{2}$ double excitations in the excited states. Going to the next-higher principal quantum number, $2 p \rightarrow 4 d$ excitation, the distribution of intensity among the main channels participator, spectator, and CI satellite decay stays roughly unchanged. A notable peculiarity on all $d$-like resonances is the weakness of $[\mathrm{Ne}] 4 p$ final states, i.e., the branching-ratio into shakeup decay channels is smaller for an outer $p$ electron than for an outer $s$ electron. Our MCDF calculations reproduce the branching ratios quantitatively for the lower resonances 4-6.

We present our measurements for the $\beta$ parameter in Table VI. For the participator decay we find a strong variation in $\beta$ with values ranging from 2 to $-0.51(5)$ as one steps through resonances $4-6$. The $\beta$ value for the $[\mathrm{Ne}] 4 \mathrm{~s}$ satellite, resolved from the $[\mathrm{Ne}] 3 d$ line, follows the same trend, as does the $[\mathrm{Ne}] 3 p$ satellite.

Figure 3 displays an enlarged section of the Auger spectra around the $[\mathrm{Ne}] 3 d$ ionic final states on resonances 2 and $4-6$. The distinct Auger lines for the pertinent $[\mathrm{Ne}] 3 d$ and $[\mathrm{Ne}] 4 s$ final states are clearly visible. The origin of these $s$ satellites following $2 p \rightarrow m d$ excitation deserves further discussion. Such satellites cannot arise from the decay of pure 
TABLE IV. Branching ratios into the different final ionic states for resonances with dominant contributions from $2 p^{-1} n d$ or double excited configurations. The designations follow Table II.

\begin{tabular}{|c|c|c|c|c|c|c|c|c|c|c|}
\hline \multirow[b]{2}{*}{$n l$} & \multirow[b]{2}{*}{$E_{b}(\mathrm{eV})$} & \multicolumn{3}{|c|}{ Res. $2\left(2 p^{-1} 3 s 3 p^{2}\right)$} & \multicolumn{4}{|c|}{ Res. $4\left(2 p_{3 / 2}^{-1} 3 d\right)$} & \multicolumn{2}{|c|}{ Res. 8a $\left(2 p_{3 / 2}^{-1} 4 d\right)$} \\
\hline & & Expt. (a) & Expt. (b) & Expt. [4] & Expt. (a) & Expt. (b) & Expt. [4] & Theor. & Expt. (b) & Theor. \\
\hline $3 s$ & 7.65 & $28.3(20)$ & 27.1(10) & $24(2)^{\mathrm{a}}$ & $83.6(30)$ & $80.8(7)$ & $83(3)$ & 84.3 & $69.9(10)$ & 59.4 \\
\hline $3 p$ & 12.07 & $26.1(20)$ & $28.6(5)$ & $28(2)$ & $5.9(5)$ & $6.6(3)$ & $6.5(3)$ & 6.2 & $14.4(4)$ & 19.2 \\
\hline $4 s$ & 16.30 & & $14.5(8)$ & & & $0.8(5)$ & & 1.6 & $1.3(4)^{\mathrm{b}}$ & 1.1 \\
\hline $3 d$ & 16.51 & $38.8(30)^{\mathrm{b}}$ & $22.9(8)$ & $42(3)^{b}$ & $8.4(5)^{\mathrm{b}}$ & $9.1(5)$ & $9.0(4)^{b}$ & 7.2 & & 0.3 \\
\hline $4 p$ & 17.64 & $2.3(4)$ & $1.7(3)$ & $2.0(4)$ & $1.2(5)$ & $1.3(1)$ & $1.1(1)$ & $0^{\mathrm{c}}$ & & \\
\hline $5 s, 4 d$ & 19.2 & $4.5(6)$ & $4.3(3)$ & $4.3(6)$ & $1.0(5)$ & $1.0(1)$ & $0.71(6)$ & 0.6 & $10.0(5)$ & 19.9 \\
\hline $5 p$ & 19.73 & & & & & & & & $<1$ & $0^{\mathrm{c}}$ \\
\hline \multirow[t]{2}{*}{$6 s, 5 d$} & 20.45 & & & & & & & & $2.9(3)$ & 0 \\
\hline & & \multicolumn{4}{|c|}{ Res. $5\left(2 p^{-1} 3 d, 2 p^{-1} 3 s 3 p^{2}\right)$} & \multicolumn{4}{|c|}{ Res. $6\left(2 p_{1 / 2}^{-1} 3 d\right)$} & $\begin{array}{c}\text { Res. } 10 \\
\left(2 p_{1 / 2}^{-1} 4 d\right) \\
\end{array}$ \\
\hline$n l$ & $E_{b}(\mathrm{eV})$ & Expt. (a) & Expt. (b) & Expt. [4] & Theor. & Expt. (a) & Expt. (b) & Expt. [4] & Theor. & Expt. (b) \\
\hline $3 s$ & 7.65 & $67.2(20)$ & $65.6(7)$ & $67(2)$ & 66.8 & $57.3(30)$ & $57.0(5)$ & $54(2)$ & 54.1 & $61.9(10)$ \\
\hline $3 p$ & 12.07 & $22.9(10)$ & $23.8(4)$ & $24(1)$ & 27.8 & $35.6(20)$ & $35.5(5)$ & $38(2)$ & 41.9 & $26.2(4)$ \\
\hline $4 s$ & 16.30 & & $0.7(3)$ & & 1.1 & & $0.7(5)$ & & 0.8 & \\
\hline $3 d$ & 16.51 & $8.1(5)^{b}$ & $7.5(3)$ & $8.0(4)^{b}$ & 3.9 & $6.3(5)^{b}$ & $5.1(5)$ & $6.2(2)^{b}$ & 2.7 & $1.9(4)$ \\
\hline $4 p$ & 17.64 & $0.8(1)$ & $0.8(1)$ & $0.74(7)$ & $0^{c}$ & & $0.5(2)$ & $0.5(2)$ & $0^{\mathrm{c}}$ & $<1$ \\
\hline $5 s, 4 d$ & 19.2 & $1.0(1)$ & $1.1(1)$ & $0.91(9)$ & 0.4 & $0.8(5)$ & $0.8(2)$ & $1.0(3)$ & 0.5 & $6.8(4)$ \\
\hline $5 p$ & 19.73 & & & & & & & 0 & & $<1$ \\
\hline $6 s, 5 d$ & 20.45 & & & & & & & $0.5(2)$ & 0 & $1.6(3)$ \\
\hline
\end{tabular}

${ }^{\mathrm{a}}$ Contributions of direct transitions has been removed.

${ }^{\mathrm{b}}$ Sum of $3 d$ and $4 s$.

${ }^{\mathrm{c}}$ Not included in calculation.

$2 p^{-1} m d$ intermediate states because of the orthogonality between the angular part of the intermediate $m d$ wave function and the final $n s$ wave function. There are essentially two mechanisms which can lead to the production of these states. The first is that the $2 p^{-1} m d$ intermediate state is not pure, but contains some admixture of the $2 p^{-1} 4 s$ state. Spectator decay of this admixed state could then lead to the appearance of the $[\mathrm{Ne}] 4 s$ line on these resonances. Another perhaps more interesting mechanism is shakeup of one of the $3 \mathrm{~s}$ electrons to a $4 s$-Rydberg orbital following participator decay of the $2 p^{-1} m d$ state. Although the overlap integral governing such a decay is likely to be small (unlike most spectator shakeup processes, the overlap integral in this case is not between 2 Rydberg orbitals) the appearance of such lines could be made possible because participator decay is so strong on these resonances. A hint as to which mechanism, if either, is dominant comes from the angular distribution of these lines. For the case where these lines arise from CI in the intermediate state, their $\beta$ values should be similar to those observed on the $2 p \rightarrow n s$ excitations. If they arise from $3 s$ shakeup accompanying participator decay, then their $\beta$ values should be the same as the participator line. An examination of Table VI and Fig. 2 indicates that these lines clearly follow the $\beta$ values of the participator lines. Furthermore, their relative intensity with respect to the $3 s$-participator line on resonances $4-6$, Table IV, is about $1 \%$ in all cases. This gives additional support to the shakeup picture. Hence, at least for these resonances, it would appear that shakeup of the $3 \mathrm{~s}$ electron during participator decay is the dominant mechanism for their production. If so, this would be the first time such a mechanism has been observed.

The theory reproduces the branching ratios for the satellites into the $[\mathrm{Ne}] 4 s$ states grossly, although no provisions have been taken to take relaxation into account. This is explained from the small mixings of several $[\mathrm{Ne}] n s$ final states with a different principal quantum number $n$ by the CI calculation we used to obtain the final state wave functions. We would not like to overinterpret our theoretical results for this case. As the energies of the final states in our CI calculation, in spite of the mixing, differ markedly from an SCF calculation, and as the pertinent mixing coefficients are very small, the agreement may well be fortuitous.

The $2 p_{1 / 2} \rightarrow 4 d$ excitation, resonance 10 , shows the smallest angular anisotropies of all considered $2 p^{-1} m d$ resonances. This supports the intuitive picture of a decrease in importance of the outer electron with higher principal quantum number. In the limit of normal Auger decay (excitation into the continuum) isotropic emission is expected for a $2 p_{1 / 2}^{-1}$ vacancy state, as it cannot be aligned. The isotropy of

TABLE V. Composition of intermediate states after $\mathrm{Mg}$ $2 p \rightarrow 3 d$ excitation according to our MCDF calculations.

\begin{tabular}{cccc}
\hline \hline $\begin{array}{c}\text { Resonance } \\
\text { No. }\end{array}$ & $2 p_{3 / 2}^{-1} 3 d$ & $2 p_{1 / 2}^{-1} 3 d$ & $2 p^{-1} 3 s 3 p^{2}$ \\
\hline 4 & 0.726 & 0 & 0.214 \\
5 & 0.354 & 0.267 & 0.292 \\
6 & 0.142 & 0.423 & 0.316 \\
\hline \hline
\end{tabular}



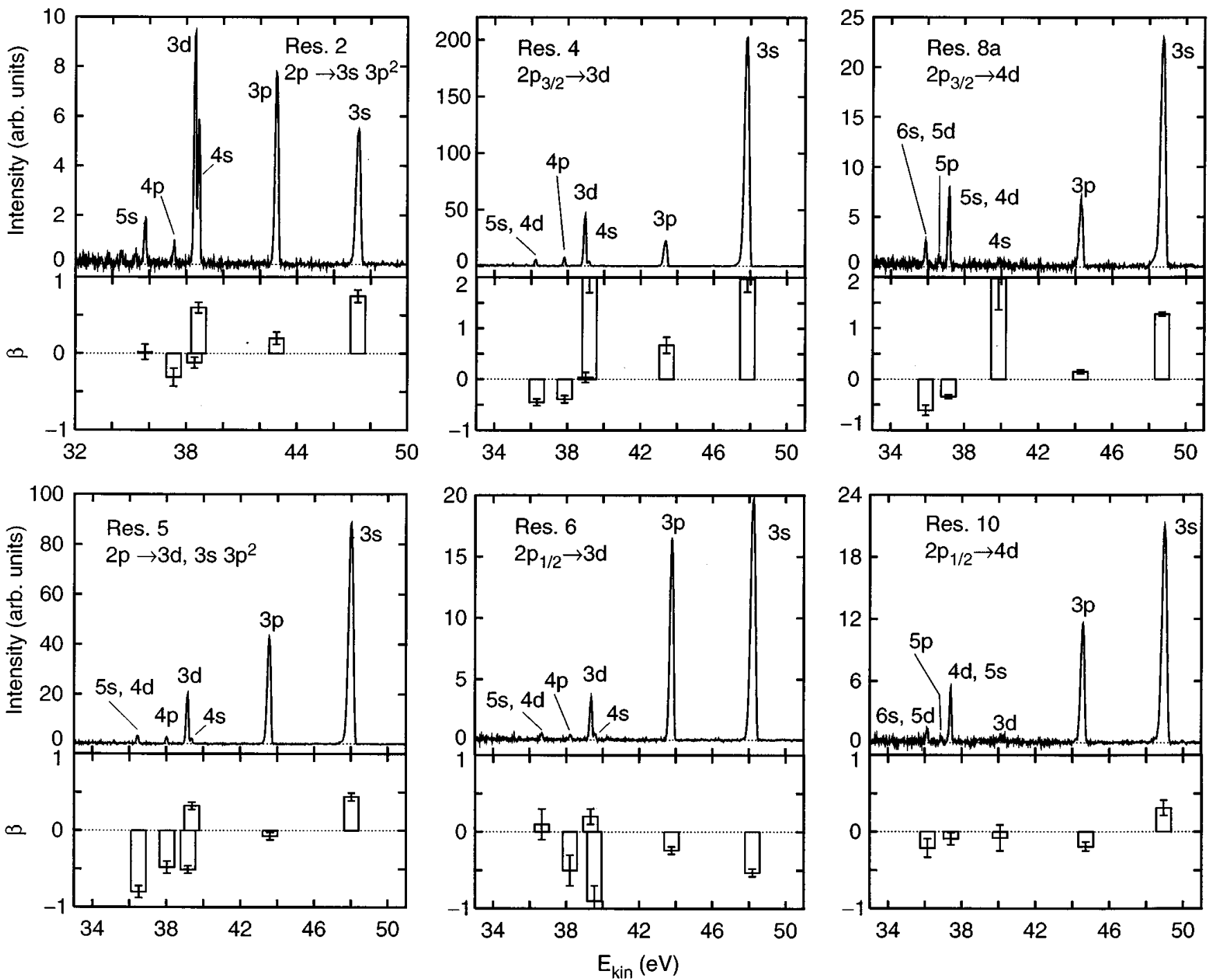

FIG. 2. Electron spectra and the $\beta$ parameter recorded on the $\mathrm{Mg} 2 p^{-1} m d$ and $2 p^{-1} 3 s 3 p^{2}$ resonances. Refer to Fig. 1 for further explanations.

the normal $\operatorname{Mg} L_{2} M M$ Auger decay has been verified experimentally [41].

We have satisfactory agreement between experiment and theory for the anisotropy of the spectator and participator lines for resonances 4-6, as already discussed in Ref. [3]. This remains true, if the $[\mathrm{Ne}] 4 s$ and $[\mathrm{Ne}] 3 d$ final ionic states are resolved. The anisotropy of the $[\mathrm{Ne}] 3 p$ channel cannot be reproduced theoretically, as discussed in the preceding subsection.

The branching ratios on resonance 2 seem to us the most intuitively puzzling. Whitfield et al. [4] and our low resolution experiment both find similar strengths for the decays into the $[\mathrm{Ne}] 3 s,[\mathrm{Ne}] 3 p$, and $[\mathrm{Ne}] 3 d, 4 s$ satellites. Our high resolution measurements indicate that on this resonance the $[\mathrm{Ne}] 4 s$ and $[\mathrm{Ne}] 3 d$ lines are populated with considerable strength. We note that the $[\mathrm{Ne}] 4 s$ line on this resonance probably cannot be explained by shakeup of the participator line. Here the relative intensity between this line and the participator peak is greater than $50 \%$, a value which is far too large to be expected from this kind of shakeup process. Hence, we must presume that resonance 2 contains a significant admixture of the $2 p^{-1} 4 s$ configuration. A $2 p^{-1} 4 s$ con- tribution to the excited state could also explain the shakeup line to $[\mathrm{Ne}] 5 s$.

Our calculations for the two levels energetically nearest to the observed resonance 2 do not reproduce the observed decay pattern. These levels are theoretically assigned as predominantly $2 p^{-1} 3 s 3 p^{2}$. Calculations of the branching ratios result in population of the participator and the $3 p$ satellite lines only. This is consistent with our calculations for resonances $4-6$, but in contradiction with the experiment.

The asymmetry parameters for the resonant Auger lines resulting in the decay of resonance 2 show a systematical difference by 0.2 between the low- and high-resolution measurements. This might stem from a small contribution of nearby resonance 3 , which was not completely separated from no. 2 in the low-resolution measurements. A small (incoherent) admixture of this resonance would influence the $\beta$ towards higher values. Further discussions will deal with the high-resolution results only.

The pattern of the resonant Auger $\beta$ parameters on resonance 2 shares similarities with the other $2 p \rightarrow m d$ resonances, especially to resonance 5 . With all resonances $4-6$, 
TABLE VI. Asymmetry parameter $\beta$ of the resonant Auger lines following $2 p \rightarrow n d$ excitation or excitation into doubly excited states. Results for resonance $4-6$ have been partly given in Ref. [3].

\begin{tabular}{|c|c|c|c|c|c|c|c|}
\hline \multirow[b]{2}{*}{$n l$} & \multicolumn{2}{|c|}{ Res. $2\left(2 p^{-1} 3 s 3 p^{2}\right)$} & \multicolumn{3}{|c|}{ Res. $4\left(2 p_{3 / 2}^{-1} 3 d\right)$} & \multicolumn{2}{|c|}{ Res. $8 \mathrm{a}\left(2 p_{3 / 2}^{-1} 4 d\right)$} \\
\hline & Expt. (a) & Expt. (b) & Expt. (a) & Expt. (b) & Theor. & Expt. (b) & Theor. \\
\hline $3 s$ & $1.06(5)$ & $0.75(8)$ & $1.90(5)$ & $1.97(27)$ & 1.98 & $1.28(4)$ & 1.27 \\
\hline $3 p$ & $0.38(5)$ & $0.20(8)$ & $0.65(5)$ & $0.67(16)$ & -0.15 & $0.15(4)$ & 0.11 \\
\hline $4 s$ & & $0.60(7)$ & & $2.00(30)$ & 1.98 & $1.98(61)^{\mathrm{b}}$ & 1.37 \\
\hline $3 d$ & $0.34(5)^{\mathrm{b}}$ & $-0.12(7)$ & $0.32(5)^{b}$ & $0.04(10)$ & -0.17 & & -0.05 \\
\hline $4 p$ & $-0.06(8)$ & $-0.31(12)$ & $-0.30(5)$ & $-0.39(7)$ & c & & \\
\hline $5 s, 4 d$ & $0.20(8)$ & $0.02(10)$ & $-0.47(5)$ & $-0.45(7)$ & 0.08 & $-0.34(4)$ & -0.42 \\
\hline $6 s, 5 d$ & & & & & & $-0.62(10)$ & $\mathrm{a}$ \\
\hline
\end{tabular}

\begin{tabular}{|c|c|c|c|c|c|c|c|}
\hline \multirow[b]{2}{*}{$n l$} & \multicolumn{3}{|c|}{ Res. $5\left(2 p^{-1} 3 d, 2 p^{-1} 3 s 3 p^{2}\right)$} & \multicolumn{3}{|c|}{ Res. $6\left(2 p_{1 / 2}^{-1} 3 d\right)$} & \multirow{2}{*}{$\frac{\text { Res. } 10\left(2 p_{1 / 2}^{-1} 4 d\right)}{\text { Expt. (b) }}$} \\
\hline & Expt. (a) & Expt. (b) & Theor. & Expt. (a) & Expt. (b) & Theor. & \\
\hline $3 s$ & $0.44(5)$ & $0.45(5)$ & 0.69 & $-0.53(5)$ & $-0.51(5)$ & -0.51 & $0.31(10)$ \\
\hline $3 p$ & $-0.07(5)$ & $-0.10(5)$ & 0.05 & $-0.24(5)$ & $-0.17(5)$ & 0.02 & $-0.19(6)$ \\
\hline $4 s$ & & $0.32(5)$ & 0.83 & & $-0.9(2)$ & -0.37 & \\
\hline $3 d$ & $-0.37(5)^{\mathrm{b}}$ & $-0.51(5)$ & -0.39 & $0.10(5)^{b}$ & $0.3(1)$ & 0.21 & $-0.08(17)^{\mathrm{b}}$ \\
\hline $4 p$ & $-0.48(8)$ & $-0.49(6)$ & c & & $-0.5(2)$ & $\mathrm{c}$ & \\
\hline $5 s, 4 d$ & $-0.80(8)$ & $-0.70(5)$ & -0.31 & $0.14(8)$ & $0.1(2)$ & -0.13 & $-0.09(8)$ \\
\hline $6 s, 5 d$ & & & & & & & $-0.21(12)$ \\
\hline
\end{tabular}

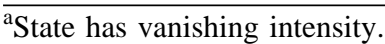

${ }^{\mathrm{b}}$ Average over $3 d$ and $4 s$.

${ }^{\mathrm{c}}$ Not included in calculation.

the $\beta$ parameters for the $[\mathrm{Ne}] 3 s$ and the $[\mathrm{Ne}] 4 s$ line on a given resonance are nearly identical, as noted earlier. This is in contrast to the $n s$ excitations, where spectator and participator decay have marked deviations in their angular anisotropy, and suggests a mixing of the $2 p^{-1} 3 d$ excited states

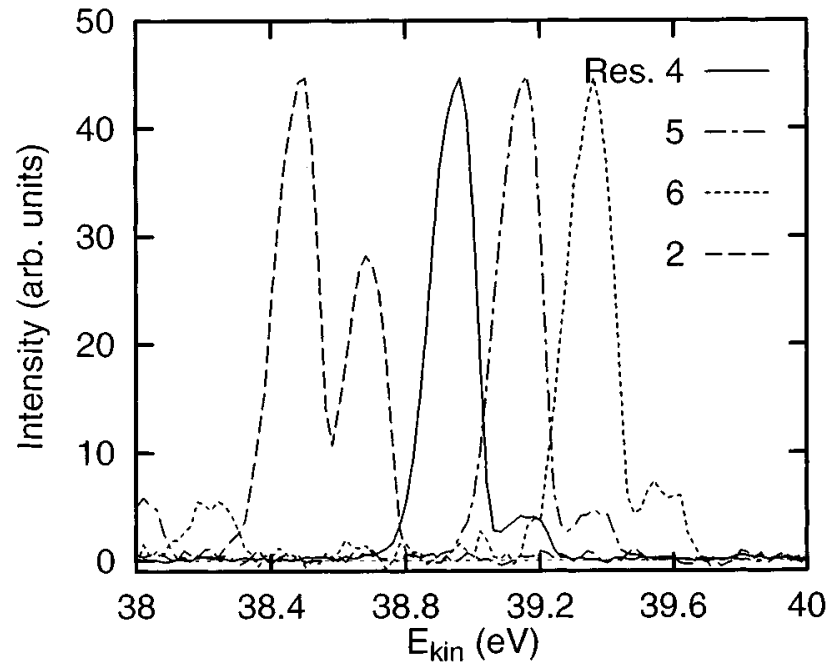

FIG. 3. Enlarged sections of the resonant Auger spectra displayed in Fig. 2. The larger peak of each spectrum is from decay to the $[\mathrm{Ne}] 3 d$ final ionic state, the smaller from decay to $[\mathrm{Ne}] 4 \mathrm{~s}$. The $y$ axis refers to the spectrum of resonance 4 . The other spectra have been scaled to equal height of the [Ne] $3 d$ peak. The scaling factors are 2.2 for resonance $5,12.7$ for resonance 6 , and 4.8 for resonance 2 . into resonance 2 . The mere existence of this resonance would be made more plausible by such a mixing. As to the 14 possible doubly excited states resulting from the $2 p^{-1} 3 s 3 p^{2}(J=1)$ configuration, only three resonances other than No. 2 have been assigned. All of these are located above the $2 p$ thresholds [4,5]. That is, most of the $2 p^{-1} 3 s 3 p^{2}(J=1)$ double excitations are not observed in the absorption spectra. On the contrary, the $2 p \rightarrow 3 d$ resonances are among the strongest of all $\mathrm{Mg} 2 p$ resonances.

\section{Interpretation and discussion}

The excited $\operatorname{Mg} 2 p^{-1} n s$ states preferentially decay via pure spectator transitions and those involving shakeup of the spectator electron. However, predictions of the anisotropy parameter by a model delineated in this work, similar to the strict spectator model of [30], are borne only by the $2 p_{1 / 2} \rightarrow 5 s$ excitation. Intuitively, one would expect increasing quality of the spectator model predictions towards excitation to higher principal quantum numbers. The transition of the resonant Auger anisotropy into the normal Auger anisotropy following core electron excitation into the orbitals of a Rydberg series was investigated numerically by Tulkki et al. [43]. These authors considered $2 p \rightarrow n s$ excitation in Ar. They compared the resonant Auger anisotropy averaged over the splitting caused by the spectator electron to the normal Ar $L_{3} M M$ Auger decay. As a result of this work, one should not expect the influence of the spectator electron to necessarily vanish quickly. For some of the final states investigated, even excitation into a $14 s$ orbital still differed by as much as $10 \%$ from the anisotropy without the presence of the spectator electron. 
All spectator transitions on the $n s$ resonances show shakeup satellites to the $[\mathrm{Ne}](n+1)$ final state. Their anisotropy parameters are indeed similar or identical to the corresponding main lines, as is predicted by shake theory. For decays to $[\mathrm{Ne}](n-1)$ final states on the $2 p \rightarrow 5 s$ resonances this is not the case. We suggest that these final states are not populated entirely by shakedown from the intermediate state with next-higher $n$.

The most important characteristic of the decay spectra on both the $3 d$ and $4 d$ resonances is the amplification of the normal $\mathrm{Mg} 3 s$ photoline. This qualitatively differentiates them from the $n s$ resonances. A hint to the interpretation of these observations is provided by a comparison with $\mathrm{Ca}$. This element resides in the Periodic Table one row below $\mathrm{Mg}$. Its electronic ground state is $[\mathrm{Ne}] 3 s^{2} 3 p^{6} 4 s^{2}$; in the following we will use the notation [Ar] $4 s^{2}$, etc. In Ref. [16] extensive studies of the $4 s$ photoionization cross section and of several satellite states along the $\mathrm{Ca} 3 p \rightarrow 3 d$ resonance are reported. The final state of direct photionization, $[\mathrm{Ar}] 4 \mathrm{~s}$, as well as the $[\mathrm{Ar}] 3 d,[\mathrm{Ar}] 4 p$, and $[\mathrm{Ar}] 5 s$ satellites are considerably enhanced at this resonance. The $3 p \rightarrow 3 d$ excitation was termed a "'giant dipole resonance.' However, the main line to satellite branching ratios undergo no marked variation along the resonance. In the interpretation of Bizau et al. [16] this is a sign for the very fast decay of the excited state via direct recombination. This mechanism, according to the authors, would enhance the photoionization main line and the satellites mixed in via ground state correlations to the same extent. This picture is not the usual two-step model of first excitation, then radiationless decay. Instead it can be viewed as photoionization enhanced via collective behavior of the $3 p$ shell. Analogously, the satellite states seen in the $\mathrm{Mg}$ case could also be mainly an indication of correlations already present within the $\mathrm{Mg}$ ground state.

In particular, the decay spectrum following excitation of resonance 4 strongly resembles the nonresonant $\mathrm{Mg} 3 \mathrm{~s}$ photoionization spectrum, as was previously noted by Whitfield et al. [4]. Investigations of the $L_{2,3}$ Auger spectrum of $\mathrm{Ca}$ found strong correlation effects between the $[\mathrm{Ar}] 4 s^{2}$, [Ar] $3 d 4 s$, and [Ar] $3 d^{2}$ states [12]. This was attributed to a "collapse" of the $3 d$ orbital due to creation of the $2 p$ vacancy. Referring to this interpretation, Whitfield et al. [4] proposed that a similar effect could also enhance the overlap between the $2 p$ and $3 d$ orbitals in $\mathrm{Mg}$. Our calculations partly confirm this assertion. The Slater integral involving participator decay, $R^{1}(3 s, 3 d ; \varepsilon l, 2 p)$, has a large value, however we found no indication of an actual collapse of the $3 d$ orbital, as discussed below.

Briefly, we can say the following about the $2 p \rightarrow m d$ excitations: (i) spectator decay plays a minor role for the $m=3,4$ excitations. This results from the large overlap of the $3 d$, and possibly also the $4 d$ orbital with the $2 p$ orbital of the intermediate state; (ii) satellite states observed on these resonances are indicative of correlations already present in the $\mathrm{Mg}$ ground state; (iii) assuming the assignment of resonance 12 as $2 p^{-1} 3 s^{2} 5 d$ and $2 p^{-1} 3 s^{2} 6 d$ excited states in Ref. [5] is correct, the decay dynamics reverts back to the dominance of spectator decay as found for the $s$-like resonances. However, the validity of this resonance assignment cannot be proven within this work.
TABLE VII. Calculated partial decay rates of the $2 p \rightarrow 4 s, 3 d$ resonances ( $\mu$ a.u., upper panel) and calculated $\beta$ parameters (lower panel). Final states are designated by the lone electron outside of the $[\mathrm{Ne}]$ core. Designations $1 \mathrm{C}, 2 \mathrm{C}$, and $14 \mathrm{C}$ refer to the size of the basis set, as explained in the text. Letters (a)-(c) refer to different mechanisms influencing the results when adding configurations to the basis set. The lower panel deviates slightly from Table I, Ref. [3] because final state mixing coefficients from a CI calculation have been used, see text.

\begin{tabular}{|c|c|c|c|c|c|c|}
\hline \multirow{2}{*}{$\begin{array}{l}\text { Excited } \\
\text { state }\end{array}$} & \multirow{2}{*}{$\begin{array}{l}\text { Final } \\
\text { state }\end{array}$} & \multicolumn{5}{|c|}{ Partial width in $\mu$ a.u. (theory) } \\
\hline & & $1 \mathrm{C}$ & & $2 \mathrm{C}$ & & $14 \mathrm{C}$ \\
\hline \multirow{10}{*}{$\begin{array}{l}\text { Res. } 1 \\
\left(2 p_{3 / 2}^{-1} 4 s\right) \\
\text { Res. } 3 \\
\left(2 p_{1 / 2}^{-1} 4 s\right) \\
\text { Res. } 4 \\
\left(2 p_{3 / 2}^{-1} 3 d\right) \\
\text { Res. } 5 \\
\left(2 p^{-1} 3 s 3 p^{2}\right) \\
\text { Res. } 6 \\
\left(2 p_{1 / 2}^{-1} 3 d\right)\end{array}$} & $3 s$ & 0.9 & (b) $\rightarrow$ & 0.3 & & 1.4 \\
\hline & $4 s$ & 5.1 & (a) $\rightarrow$ & 11.9 & $(\mathrm{a}) \rightarrow$ & 11.1 \\
\hline & $3 s$ & 0.9 & (b) $\rightarrow$ & 0.5 & & 4.4 \\
\hline & $4 s$ & 4.3 & (a) $\rightarrow$ & 9.7 & (a) $\rightarrow$ & 10.5 \\
\hline & $3 s$ & 2.8 & & 34.3 & & 117.3 \\
\hline & $3 d$ & 4.0 & & 1.4 & & 10.0 \\
\hline & $3 s$ & 9.7 & & 103.2 & $(\mathrm{c}) \rightarrow$ & 177.0 \\
\hline & $3 d$ & 4.0 & & 1.4 & & 10.2 \\
\hline & $3 s$ & 5.3 & & 50.8 & $(\mathrm{c}) \rightarrow$ & 198.6 \\
\hline & $3 d$ & 3.4 & & 1.0 & & 9.8 \\
\hline & & \multicolumn{5}{|c|}{$\beta$ (theory) } \\
\hline \multirow[t]{2}{*}{ Res. 1} & $3 s$ & 0.1 & & -1.0 & & -0.2 \\
\hline & $4 s$ & 0.9 & & 0.6 & & 0.8 \\
\hline \multirow[t]{2}{*}{ Res. 3} & $3 s$ & -0.5 & & -1.0 & & -0.8 \\
\hline & $4 s$ & 0.2 & & 0.4 & & 0.3 \\
\hline \multirow[t]{2}{*}{ Res. 4} & $3 s$ & 1.8 & & 1.9 & & 2.0 \\
\hline & $3 d$ & -0.2 & & -0.3 & & 0.2 \\
\hline \multirow[t]{2}{*}{ Res. 5} & $3 s$ & 2.0 & & -0.7 & & 0.7 \\
\hline & $3 d$ & -0.3 & & -0.4 & & -0.4 \\
\hline \multirow[t]{2}{*}{ Res. 6} & $3 s$ & 2.0 & & 0.5 & & -0.5 \\
\hline & $3 d$ & 0.0 & & 0.1 & & 0.2 \\
\hline
\end{tabular}

\section{THEORETICAL ANALYSIS}

So far, we have only discussed theoretical results obtained with the largest basis set $14 \mathrm{C}$. To further elucidate the influence of the configuration space, we show the partial decay widths of the participator and spectator decay channels and their anisotropy parameters $\beta$ for the resonances 1,3 , and 4-6 in Table VII. Results are given for all three basis sets employed in our calculations. The partial decay width is proportional to the intensity of an autoionization line. Its behavior upon adding more configurations can give hints to the underlying mechanisms responsible for changes within other quantities as well. In particular, we refer to the changes in the calculated $\beta$ parameter.

The main mechanisms responsible for the drastic changes within the calculated partial widths and the $\beta$ parameters are designated by letters $(a-c)$ in Table VII. Along $(a)$ and (b) the $3 p^{2}$ configurations are added to the intermediate state. For the spectator decay of $s$-Rydberg states this leads to an increase of the partial width by a factor of 2 , along with an enhanced mixing of $2 p_{3 / 2}^{-1}$ and $2 p_{1 / 2}^{-1}$ vacancy states. This enormous influence of a relatively small admixture of a different configuration was traced back to the kinetic energy dependence of the pertinent Slater integrals in Ref. [3]. Al- 
TABLE VIII. Mixing coefficients, Slater integrals, and angular momentum coefficients [36] of the transition amplitudes for spectator decay following excitation to resonance 1 . Results were calculated using the $14 \mathrm{C}$ basis set. The Slater integrals and partial transition amplitudes are given in $10^{-3}$ a.u.

\begin{tabular}{ccccccc}
\hline \hline & $\begin{array}{c}\text { Excited } \\
\text { state }\end{array}$ & $\begin{array}{c}\text { Mixing } \\
\text { coefficient }\end{array}$ & $\begin{array}{c}\text { Partial } \\
\text { wave }\end{array}$ & $\begin{array}{c}\text { Slater } \\
\text { integral }\end{array}$ & $\begin{array}{c}\text { Ang. } \\
\text { coeff. }\end{array}$ & Amplitude \\
\hline $2 p_{3 / 2}^{-1}$ & $3 s^{2} 4 s$ & 0.9432 & $p_{3 / 2}$ & -2.187 & 0.333 & -0.687 \\
& $\left(3 p_{3 / 2}^{2}\right)_{0} 4 s$ & 0.1809 & & 5.693 & -0.707 & -0.728 \\
& $\left(3 p_{1 / 2}^{2}\right)_{0} 4 s$ & 0.1409 & & -0.437 & -0.200 & 0.012 \\
\hline $2 p_{1 / 2}^{-1}$ & $3 s^{2} 4 s$ & 0.0847 & $p_{1 / 2}$ & -1.945 & -0.333 & 0.055 \\
& $\left(3 p_{3 / 2}^{2}\right)_{0} 4 s$ & 0.0179 & & -0.093 & 0.283 & -0.0005 \\
& $\left(3 p_{1 / 2}^{2}\right)_{0} 4 s$ & 0.0098 & & 5.989 & 1.000 & 0.059 \\
\hline \hline
\end{tabular}

though the observed kinetic energy of an autoionization or Auger line is fixed, we can numerically treat off-shell transition amplitudes belonging to a nonphysical transition energy as well. By doing so we found a sign change of the leading configuration's Slater integral very near the observed kinetic energy, thus enhancing the relative importance of admixed configurations.

A more quantitative analysis of these findings is shown in Table VIII. In the third column of this table the mixing coefficients of the most important configurations for the intermediate state of resonance $1\left(2 p_{3 / 2} \rightarrow 4 s\right)$ are indicated. These are the coefficients in the basis expansion of the eigenvector. The sum of the squared mixing coefficients of the pertinent relativistic configurations was referred to as the weight in Tables I and V. Clearly here the dominant configuration is the $2 p_{3 / 2}^{-1} 3 s^{2} 4 s$. The most important admixture involving a $2 p_{1 / 2}$ hole is the $2 p_{1 / 2}^{-1} 3 s^{2} 4 s$ configuration. Compared to it, the mixing coefficients of the $2 p_{1 / 2}^{-1} 3 p^{2} 4 s$ states seem small, namely 0.0179 and 0.0098 . However, the Slater integral $R^{0}\left(3 p_{j}, 3 p_{j} ; 2 p_{j}, \varepsilon p_{j}\right)$ responsible for the decay of the $2 p^{-1} 3 p^{2} 4 s$ configurations to a $[\mathrm{Ne}] 4 s$ final ionic state has a much larger value for a given $j$ than the $R^{1}\left(3 s, 3 s ; 2 p_{j}, \varepsilon p_{j}\right)$ Slater integral, governing the spectator decay of the $2 p^{-1} 3 s^{2} 4 s$ configurations (column 5). Moreover, the former Slater integral has rank $\kappa=0$, the latter $\kappa=1$. Therefore, the angular momentum coefficient of the decay amplitude produced by the former for a given $j$ is larger as well, a factor of 6 for $j=3 / 2$, and a factor of 9 for $j=1 / 2$ (square of column 6). (By angular momentum coefficient we mean the part of the decay amplitude resulting from angular integration and from coupling and antisymmetrization of the single-particle orbitals [36].) This entails that the product of the mixing coefficient, Slater integral, and angular momentum coefficient (rightmost column) for one of the $2 p_{1 / 2}^{-1} 3 p^{2} 4 s$ admixtures is larger than for $2 p_{1 / 2}^{-1} 3 s^{2} 4 s$. Its value reaches about $9 \%$ of the leading term's amplitude. Due to an interference term between the $\varepsilon p_{3 / 2}$ and $\varepsilon p_{1 / 2}$ partial waves in the expression for $\alpha_{2}$, Eq. (5), such seemingly small admixtures can have a pronounced influence on the anisotropy parameter. We note that the increase in partial widths along $(a)$ going to the $2 \mathrm{C}$ basis set is due to the admixtures of $2 p_{3 / 2}^{-1} 3 p^{2} 4 s$, which enhance the amplitude by the same mechanism, but have no influence on the value of $\beta$.

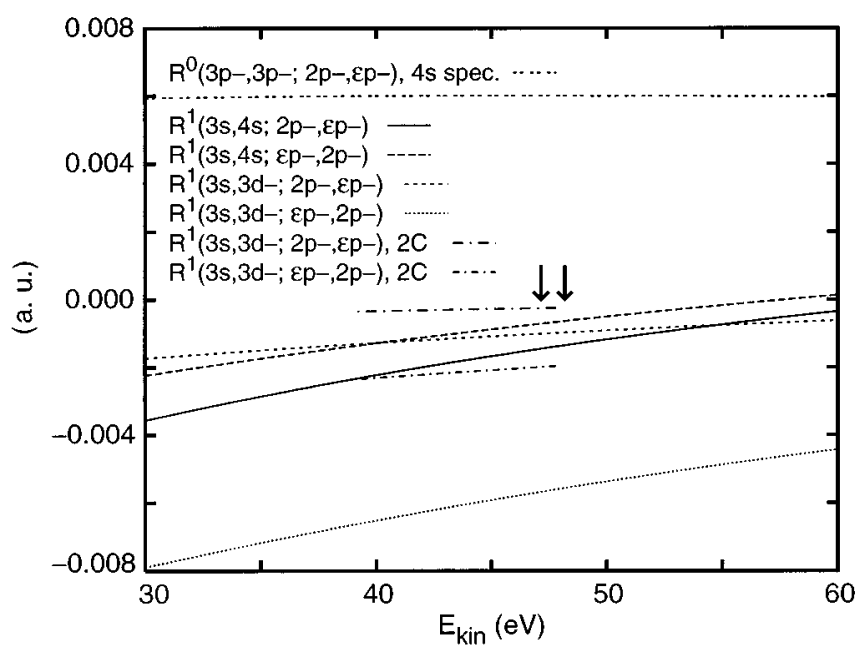

FIG. 4. Slater integrals for participator decay of the $2 p^{-1} 3 s^{2} 4 s$ and $2 p^{-1} 3 s^{2} 3 d$ excited states as a function of the kinetic energy of the continuum electron. The left (right) arrow marks the experimental kinetic energy of the $3 s$ participator line on resonance 1 (6). A trailing minus sign designates spin-down coupling of the orbital, i.e., $3 d \equiv 3 d_{5 / 2}, 3 d-\equiv 3 d_{3 / 2}$. The $y$ axis is in atomic units.

For the participator decay the calculated decay width does not increase upon addition of the $3 p^{2} 4 s$ configuration (along $b)$. For the amplitude of this decay two Slater integrals contribute: the direct integral $R^{1}\left(3 s, 4 s ; 2 p_{j}, \varepsilon p_{j}\right)$ and the exchange integral $R^{1}\left(3 s, 4 s ; \varepsilon p_{j}, 2 p_{j}\right)$. The values of these integrals change significantly when the $3 p$ orbital is included in the variation procedure. This occurs because orthogonality between the $\varepsilon p$ continuum wave function and the $3 p$ wave function is now required. As a result, the values of both integrals diminish. However, the ratio between both integrals belonging to the same $j$ and the ratio between $j=1 / 2$ and $j=3 / 2$ emission changes as well. This leads to the changes of the participator $\beta$ along $(b)$.

Changes in the $2 p^{-1} 3 s^{2} 3 d$ decay parameters by extending the basis set from $1 \mathrm{C}$ to $2 \mathrm{C}$ can be traced back to effects similar to those in the $2 p_{3 / 2} \rightarrow 4 s$ case. By taking the $3 s 3 p^{2}$ configuration into account, the decay rate into the $[\mathrm{Ne}] 3 \mathrm{~s}$ final ionic state increases, while the orthogonalization of the $\varepsilon p$ with the $3 p$ wave function leads to a diminished spectator rate. For resonances 4 and 5 the $1 \mathrm{C}$ column in Table VII gives the results for the fine-structure states $\left[2 p_{3 / 2}^{-1}, 3 d_{3 / 2,5 / 2}\right]_{1}$, although the calculated fine-structure splitting is much smaller than the energetical difference between the two resonances. Extending the basis set further from $2 \mathrm{C}$ to $14 \mathrm{C}$, another effect designated by $(c)$ comes into play. As discussed in Ref. [3], the calculated $3 d_{j}$ orbitals are contracted towards the ionic core once a $4 d$ bound state orbital is present in the calculation. For the $3 d_{5 / 2}\left(3 d_{3 / 2}\right)$ wave function the mean radius, $\langle r\rangle$, changes from 9.39(9.43) a.u. to 6.30(6.15) a.u. In the ground state the values of $\langle r\rangle$ are $7.10(6.96)$ a.u. Thus, the reaction of the $3 d$ orbital upon creation of a $2 p$ hole cannot really be called a "collapse."

Figure 4 indicates the impact of the $3 d$ orbital's shape on the calculated values of the slater integrals. The decisive factor in the increase of the decay rate along $(c)$ is the obvious increase of the $R^{1}(3 s, 3 d ; \varepsilon p, 2 p)$ exchange integral in abso- 
lute value. This Slater integral has much larger values than $R^{1}(3 s, 4 s ; 2 p, \varepsilon p)$ and its exchange integral. These results clearly explain the dominance of participator transitions for decay of the $2 p^{-1} 3 s^{2} 3 d$ excited states, and their relative weakness for the $2 p^{-1} 3 s^{2} 4 s$ excited states.

\section{CONCLUSION}

We have presented a systematic study of the angular anisotropy of resonant Auger decay of $2 p$-photoexcited $\mathrm{Mg}$ principally along four Rydberg series which could be differentiated in their character as either $2 p^{-1} 3 s^{2} n s$ or $2 p^{-1} 3 s^{2} m d$. This is the first time, to our knowledge, that such isolation of the higher lying states, $n=5$ and $m=4$, in an emission experiment on $\mathrm{Mg}$ has been acheived, owing to the use of a high resolution high flux monochromator. For $2 p \rightarrow n s$ excitations we find spectator decay, including shakeup and shakedown, to be the dominant decay mode. Deviations of the angular anisotropy of the lower lying $2 p \rightarrow n s$ excitations from simple predictions are due to the mixing of the $2 p_{1 / 2}$ and the $2 p_{3 / 2}$ core holes in the presence of the excited electron. Our experimental results for one higher resonance excitation, namely the $2 p_{1 / 2} \rightarrow 5 s$, is suggestive of the decay dynamics returning to the values yielded by a simple application of the spectator model. Unfortunately, we lack the resolution in the monochromator to isolate the higher lying $n s$ Rydberg series from nearby $m d$ Rydberg series. Therefore, we cannot verify the return to the spectator model behavior completely.

In sharp contrast to the $2 p \rightarrow n s$ excitations, the $2 p \rightarrow m d$ excitations are dominated by participator decay of the excited electron, with spectator decay being surprisingly weak. This indicates that one cannot always presume that spectator decay will be the dominant decay mode for the lower lying Rydberg excitations. In addition, we also observe a small degree of population in the $[\mathrm{Ne}] 4 s$ line on the $2 p \rightarrow 3 d$ excitations. This line can only arise from configuration interaction in the excited intermediate state, or from shakeup of the $3 s$ electron during participator decay. The latter interpretation is supported by the experimental observations that the angular anisotropy of this line is nearly identical to the $3 s$ main line on these resonances. If this is the dominant mode of production for this line, it would be the first time that such a mechanism has been observed.

In contrast to earlier works on noble gases [38], our MCDF calculations show a remarkable influence of configuration interaction within the intermediate state. This effect results partly from a change of sign in the leading configuration's Slater integral.

We discussed several calculational effects present in atomic CI calculations. Of special importance were differences in the orbital shape due to additional orthogonalization necessary in a larger basis set. This underlines the importance of reproducible methods to generate the basis functions for not only molecular, but also atomic CI calculations.

\section{ACKNOWLEDGMENTS}

U.H. acknowledges a grant and travel allowances from the senate of Berlin, S.B.W. is grateful for financial support from the Alexander von Humboldt Foundation, and N.M.K. for support from the Fritz-Haber-Institut. This work was also supported by the Bundesminister für Forschung und Technologie, the Deutsche Forschungsgemeinschaft, and the Academy of Finland.
[1] H. Aksela, J. Electron Spectrosc. Relat. Phenom. 72, 235 (1995).

[2] H. Aksela, S. Aksela, and N. M. Kabachnik, in VUV and Soft $X$-Ray Photoionization Studies, edited by U. Becker and D. A. Shirley (Plenum Press, New York, 1996), Chap. 11, p. 401.

[3] S. B. Whitfield, U. Hergenhahn, N. M. Kabachnik, B. Langer, J. Tulkki, and U. Becker, Phys. Rev. A 50, R3569 (1994).

[4] S. B. Whitfield, C. D. Caldwell, and M. O. Krause, Phys. Rev. A 43, 2338 (1991).

[5] G. H. Newsom, Astrophys. J. 166, 243 (1971).

[6] J. M. Esteva and G. Mehlman, Astrophys. J. 193, 747 (1974).

[7] D. L. Ederer, T. B. Lucatorto, and G. Mehlman, J. Opt. Soc. Am. 69, 520 (1979).

[8] B. Breuckmann, V. Schmidt, and W. Schmitz, J. Phys. B 9, 3037 (1976); V. Pejčev, T. W. Ottley, D. Rassi, and K. J. Ross, ibid. 10, 2389 (1977).

[9] Z. Altun, Phys. Rev. A 40, 4968 (1989).

[10] S. B. Whitfield, J. Tulkki, and T. Åberg, Phys. Rev. A 44, R6983 (1991).

[11] G. B. Armen, J. Phys. B 29, 677 (1996).

[12] W. Weber, B. Breuckmann, R. Huster, W. Menzel, W. Mehlhorn, M. H. Chen, and K. G. Dyall, J. Electron Spectrosc. Relat. Phenom. 47, 105 (1988).
[13] M. H. Chen, W. Weber, and W. Mehlhorn, J. Electron Spectrosc. Relat. Phenom. 49, 77 (1989).

[14] M. Meyer, E. v. Raven, M. Richter, B. Sonntag, R. D. Cowan, and J. E. Hansen, Phys. Rev. A 39, 4319 (1989).

[15] M. Meyer, E. v. Raven, B. Sonntag, and J. E. Hansen, Phys. Rev. A 49, 3685 (1994).

[16] J. M. Bizau, P. Gérard, F. J. Wuilleumier, and G. Wendin, Phys. Rev. A 36, 1220 (1987), and references therein.

[17] A. Yagishita, S. Aksela, Th. Prescher, M. Meyer, M. Richter, E. v. Raven, and B. Sonntag, J. Phys. B 21, 945 (1988); J. Jiménez-Mier, C. D. Caldwell, M. G. Flemming, S. B. Whitfield, and P. van der Meulen, Phys. Rev. A 48, 442 (1993).

[18] K. Ueda, J. B. West, K. J. Ross, H. Hamdy, H. J. Beyer, and H. Kleinpoppen, Phys. Rev. A 48, R863 (1993).

[19] K. Ueda, J. B. West, K. J. Ross, H. J. Beyer, H. Hamdy, and H. Kleinpoppen, J. Phys. B 26, L347 (1993).

[20] K. Ueda, J. B. West, N. M. Kabachnik, Y. Sato, K. J. Ross, H. J. Beyer, H. Hamdy, and H. Kleinpoppen, Phys. Rev. A 54, 490 (1996).

[21] U. Becker, D. Szostak, H. G. Kerkhoff, M. Kupsch, B. Langer, R. Wehlitz, A. Yagishita, and T. A. Hayaishi, Phys. Rev. A 39, 3902 (1989).

[22] K. J. Ross and B. Sonntag, Rev. Sci. Instrum. 66, 4409 (1995). 
[23] W. Peatman, C. Carbone, W. Gudat, W. Heinen, P. Kuske, J. Pflüger, F. Schäfers, and W. Schroeter, Rev. Sci. Instrum. 60, 1445 (1989).

[24] C. U. S. Larsson, A. Beutler, O. Björneholm, F. Federmann, U. Hahn, A. Rieck, S. Verbin, and Th. Möller, Nucl. Instrum. Methods A 337, 603 (1994).

[25] F. J. Wuilleumier and M. O. Krause, J. Electron Spectrosc. Relat. Phenom. 15, 15 (1979).

[26] J. M. Bizau and F. J. Wuilleumier, J. Electron Spectrosc. Relat. Phenom. 71, 205 (1995).

[27] E. G. Berezhko and N. M. Kabachnik, J. Phys. B 10, 2467 (1977).

[28] N. M. Kabachnik and I. P. Sazhina, J. Phys. B 17, 1335 (1984).

[29] W. Kronast, R. Huster, and W. Mehlhorn, Z. Phys. D 2, 285 (1986).

[30] U. Hergenhahn, B. Lohmann, N. M. Kabachnik, and U. Becker, J. Phys. B 26, L117 (1993).

[31] B. Kämmerling, B. Krässig, and V. Schmidt, J. Phys. B 23, 4487 (1990).

[32] B. Lohmann, J. Phys. B 24, 861 (1991).
[33] H. Aksela, S. Aksela, H. Pulkkinen, and A. Yagishita, Phys. Rev. A 40, 6275 (1989).

[34] J. Tulkki and A. Mäntykenttä, Phys. Rev. A 47, 2995 (1993).

[35] U. Hergenhahn, N. M. Kabachnik, and B. Lohmann, J. Phys. B 24, 4759 (1991).

[36] I. P. Grant, B. J. McKenzie, P. H. Norrington, D. F. Mayers, and N. C. Pyper, Comput. Phys. Commun. 21, 207 (1980).

[37] J. Tulkki, T. Åberg, A. Mäntykenttä, and H. Aksela, Phys. Rev. A 46, 1357 (1992).

[38] J. Tulkki, H. Aksela, and N. M. Kabachnik, Phys. Rev. A 50, 2366 (1994).

[39] N. M. Kabachnik and I. P. Sazhina, J. Phys. B 9, 1681 (1976).

[40] T. A. Carlson, D. R. Mullins, C. E. Beall, B. W. Yates, J. W. Taylor, D. W. Lindle, and F. A. Grimm, Phys. Rev. A 39, 1170 (1989); C. D. Caldwell and S. Hallman, ibid. 53, 3344 (1996).

[41] B. Kämmerling, A. Hausmann, J. Läuger, and V. Schmidt, J. Phys. B 25, 4773 (1992).

[42] Ch. E. Moore, Atomic Energy Levels, Natl. Bur. Stand. (U.S.) Circ. No. 467 (U.S. GPO, Washington D.C., 1949).

[43] J. Tulkki, H. Aksela, and N. M. Kabachnik, Phys. Rev. A 48, 2957 (1993). 